\author{
S. Weber-Carstens \\ H. Lohbrunner \\ U. Kaisers
}

\section{Lungenprotektive Strategien zur Therapie des ARDS*}

\section{Lung protective strategies in treatment of ARDS}

Summary Recognition of the potential for ventilator-associated lung injury in patients with acute respiratory distress syndrome (ARDS) has stimulated the debate on the appropriate strategies of mechanical ventilation in these patients. This review summarizes new aspects of research on ventilator-associated lung injury and provides an overview of recent prospective, randomized, controlled trials. Methods and principles guid-

Eingegangen: 11. Juli 1999

Akzeptiert: 11. August 1999

* Herrn Prof. Dr. med. Konrad J. Falke zum 60. Geburtstag gewidmet

Teilweise gefördert durch die DFG (Ka 1212/3-1)

S. Weber-Carstens $\cdot H$. Lohbrunner PD Dr. U. Kaisers (殴)

Charité, Campus Virchow-Klinikum

Medizinische Fakultät der

Humboldt-Universität zu Berlin

Augustenburger Platz 1

D-13353 Berlin

e-mail: udo.kaisers@ charite.de ing ventilatory support like pressurelimited mechanical ventilation with low tidal volumes and high levels of positive end-expiratory pressure (PEEP) as well as adjunctive, nonventilatory therapies like inhaled nitric oxide (NO) or administration of exogenous surfactant are discussed.

Key words ARDS - ventilator induced lung injury - shear stress barotrauma - volutrauma mechanotransduction - proinflammatory cytokines - lung protective ventilation - PEEP - PIP - pressure/ volume relationship - pressure controlled ventilation - NO - surfactant partial liquid ventilation - high frequency ventilation - tracheal gas insufflation - permissive hypercapnia - prone positioning

\section{Zusammenfassung Gegenstand dieser Übersicht ist die kritische Darstellung verschiedener Aspekte der bisherigen Forschung zum beat- mungsinduzierten Lungenschaden. Im Einzelnen werden die verschiede- nen Pathomechanismen, die zum be- atmungsinduzierten Lungenschaden führen können, wie z. B. ,,mechani-}

scher Scher-Stress" oder die Freisetzung proinflammatorischer Zytokine dargestellt. Ergebnisse prospektivkontrollierter, randomisierter Studien zu den Effekten kontrollierter Beatmung auf die Progression eines Lungenschadens sowie die daraus resultierenden, klinischen Empfehlungen für die Beatmungsstrategien bei Patienten mit schwerem, akuten Lungenversagen werden als Übersicht aufgezeigt. Darüber hinaus wird der Stellenwert adjuvanter Therapiemaßnahmen, wie der Inhalation von Stickstoffmonoxid oder der Applikation von exogenem Surfactant zur Lungenprotektion erörtert.

Schlüsselwörter ARDS - beatmungsinduzierter Lungenschaden shear stress - Barotrauma Volutrauma - Mechanotransduktion - proinflammatorische Zytokine lungenprotektive Beatmung PEEP - PIP - Druck-/Volumenbeziehung - druckkontrollierte Beatmung - NO - Surfactant - Partial Liquid

Ventilation - High Frequency Ventilation - Tracheale Gasinsufflation - Permissive Hyperkapnie Bauchlage

\section{Beatmungsinduzierter Lungenschaden - Hintergrund und Pathophysiologie}

Die Letalität des akuten Lungenversagens (acute respiratory distress syndrom = ARDS), das durch eine anhaltende, ausgeprägte Störung des pulmonalen Gasaustausches, ei- nen extremen Abfall der Lungencompliance und der Entwicklung eines radiologisch faßbaren interstitiellen und alveolären Lungenödems charakterisiert ist [7,9], wird auch heute noch mit über 50\% angegeben $[62,111]$.

Das ARDS beschreibt ein Syndrom heterogener Ätiopathogenese, das zu Schäden an der alveolokapillären Ein- 
heit mit konsekutivem Permeabilitätsödem [60] und Störung des endogenen Surfactantsystems führt [69]. Für die Beeinträchtigung des Surfactantsystems sind vor allem drei Mechanismen verantwortlich gemacht worden: 1) der Verlust von Surfactant in die kleinen Atemwege infolge einer überproportionalen end-exspiratorischen Radiusabnahme der Alveole; 2) die Konversion oberflächenaktiver großer Surfactantaggregate in inaktive, kleine Aggregate und 3) die Inaktivierung von Surfactant durch proteinreiche intraalveoläre Ödemflüssigkeit [46, 69]. Eine infolge der Surfactantdysfunktion erhöhte Oberflächenspannung induziert die Zunahme von Kräften, die an der Luft-Flüssigkeitsgrenzschicht der Alveole wirksam sind und führt schließlich zu end-exspiratorischem Kollaps, Atelektasen, intrapulmonalem Rechts-Links-Shunt und Hypoxämie.

Einer der Gründe für die hohe Letalität des akuten Lungenversagens wird, außer in dem durch die Grundkrankheit bestimmten deletären Verlauf einiger spezieller Formen des ARDS, auch darin gesehen, daß die zur Aufrechterhaltung annähernd normaler Blutgase notwendige, sehr aggressive Beatmung, also die Therapie selbst, zur Progression des bestehenden Lungenschadens beiträgt [37, 70, 118].

Gegenstand dieser Übersicht ist die kritische Darstellung einiger Aspekte der bisherigen Forschung zum beatmungsinduzierten Lungenschaden und die daraus resultierenden, klinischen Empfehlungen für die Beatmungsstrategien bei Patienten mit schwerem, akuten Lungenversagen.

\section{Beatmungsinduzierter Lungenschaden}

Es ist lange bekannt, daß maschinelle Beatmung einen bestehenden Lungenschaden aggravieren und damit einen eigenständigen pathogenetischen Faktor beim akuten Lungenversagen darstellen kann. Schwierigkeiten bereitet allerdings häufig die Abgrenzung des beatmungsinduzierten Lungenschadens von einer bloßen Progression der Grunderkrankung. Tierexperimentell ist überzeugend dargelegt worden, daß maschinelle Beatmung einen bestehenden parenchymatösen Lungenschaden nicht nur verschlimmern kann, sondern daß bestimmte Beatmungsformen auch eine de novo Schädigung der Lunge induzieren können.

Beatmungsinduzierte Lungenschäden werden gewöhnlich unter dem Begriff „Barotraumata“ subsummiert. Das Auftreten von extraalveolärer Luft gilt dafür als pathognomonisch. Als primärer Ort eines beatmungsinduzierten „mechanischen Stress“ wird das Endothel der alveolokapillären Einheit angenommen, mit allerdings schneller Progression des Schadens zum Epithel [25]. Der beatmungsinduzierte Lungenschaden scheint dabei weniger von der absoluten Höhe des applizierten Atemwegspitzendruckes, als vielmehr vom damit generierten Tidalvo- lumen und der Größe des end-exspiratorischen Lungenvolumens abzuhängen [25, 27]. Verschiedene Arbeitsgruppen haben interstitiell wirksame Scherkräfte durch eine zyklische Eröffnung kleiner Atemwege und Alveolen während der Inspiration mit folgendem Kollaps zum Ende der Exspiration für die Progression des Lungenschadens unter maschineller Beatmung verantwortlich gemacht [35, 49, 105].

Klinisch werden unterschiedliche Schweregrade des beatmungsinduzierten Lungenschadens bis hin zum Spannungspneumothorax beobachtet. Jüngeren Datums ist das Konzept des „Volutraumas“ infolge maschineller Beatmung mit hohen Tidalvolumina, welches prinzipiell zu den gleichen pathologischen Veränderung wie das „Barotrauma“ führen kann. Zusätzlich werden unter „Volutrauma“ jedoch auch Änderungen der Gefäßpermeabilität mit Progression des Lungenödems infolge maschineller Beatmung mit hohen Tidalvolumina verstanden.

Neben den makroskopisch faßbaren Lungenschäden treten unter maschineller Beatmung mit hohen Inspirationsdrücken und großen Tidalvolumina eine Vielzahl pathophysiologischer und ultrastruktureller Veränderungen auf.

Bereits in den siebziger Jahren waren es Webb und Tierney [133], die experimentell zeigen konnten, daß eine maschinelle Beatmung von lungengesunden Ratten bereits nach kurzer Zeit zu einem pulmonalen Ödem führt. Das Ausmaß des Lungenödems war dabei von der Höhe des applizierten Spitzendrucks abhängig: Bei einem Atemwegspitzendruck von $45 \mathrm{cmH}_{2} \mathrm{O}$ kam es zur raschen Entwicklung eines profusen alveolären Ödems, während die Beatmung mit $30 \mathrm{cmH}_{2} \mathrm{O}$ nur zu einem mäßiggradigen interstitiellen Ödem führte. Dreyfuss und Mitarbeiter [25, 28] bestätigten diese Befunde. Bei lungengesunden Ratten kam es bereits nach wenigen Minuten IPPV Beatmung zu einem Anstieg des extravaskulären Lungenwassers.

Bei größeren Tieren ist hingegen eine längere maschinelle Beatmung erforderlich, um vergleichbare Schädigungen zu erzeugen. So fanden Kolobow et al. [61] bei gesunden Schafen, die für bis zu 48 Stunden mit einem Atemwegspitzendruck von $50 \mathrm{cmH}_{2} \mathrm{O}$ beatmet wurden, ausgeprägte pulmonale Flüssigkeitseinlagerungen und Atelektasen.

Webb und Tierney [133] hatten angenommen, daß unter maschineller Beatmung eine Zunahme der transkapillären Flüssigkeitsfiltration für die Entwicklung eines Lungenödems ursächlich sei. Besonders bei Applikation hoher Spitzendrücke in Verbindung mit einem nierdrigen PEEP kann es infolge einer Inaktivierung von Surfactant zu einer Zunahme der alveolären Oberflächenspannung und damit zu einem Anstieg des kapillären, transmuralen Druckes kommen. Andere Untersucher haben diese Hypothese experimentell bestätigen können $[1,11]$. Bereits seit den sechziger Jahren ist bekannt, daß maschinelle Beatmung darüber hinaus gravierende Effekte auf extraalveoläre Gefäße hat: Während der Inspiration kommt es in- 
folge Lungendehnung zu einer Druckabnahme im perivaskulären Raum mit Dilatation extraalveolärer Gefäße. Benjamin und Mitarbeiter [8] haben gezeigt, daß es in extraalveolären Gefäßen pro $1 \mathrm{cmH}_{2} \mathrm{O}$ Anstieg des transpulmonalen Druckes zu einem Anstieg des transmuralen Druckes von bis zu $2 \mathrm{cmH}_{2} \mathrm{O}$ kommt.

Für die Entwicklung eines beatmungsinduzierten pulmonalen Ödems spielen neben der Zunahme der transkapillären Flüssigkeitsfiltration auch Änderungen der epithelialen und endothelialen Permeabilität eine entscheidende Rolle. Tierexperimentelle wie klinische Untersuchungen haben nachweisen können, daß große Lungenvolumina wie sie unter maschineller Beatmung mit hohen Drücken vorkommen, zu einer Zunahme der alveolo-epithelialen Permeabilität führen [20, 86]. Ursächlich könnten fokale Schwellungen und Zell-Lysen von Typ I-Zellen sein, wie sie in einer Untersuchung von Dreyfuss und Mitarbeitern beobachtet wurden [25].

Die Arbeitsgruppe von Lachmann [126] zeigte tierexperimentell, daß nach intratrachealer Inokulation von 100 $\mu 1$ einer 3,5-5×10 $0^{5}$ kolonieformender Einheiten Klebsiella pneumoniae pro $100 \mathrm{ml}$ enthaltenden Lösung die Translokation, d.h. der Nachweis einer systemischen Bakteriämie, von den applizierten Atemwegsdrücken abhängt: Bei den Tieren, die mit hohen Atemwegspitzendrücken aber ohne PEEP beatmet worden waren, fand sich eine signifikante Zunahme der Bakteriämie mit Klebsiella pneumoniae. Die Autoren nehmen an, daß während der Beatmung mit hohen Spitzendrücken $\left(30 \mathrm{cmH}_{2} \mathrm{O}\right)$ und ZEEP eine Progression des Lungenödems mit Verminderung der bakteriziden Potenz von Alveolarmakrophagen sowie einer Inaktivierung von Surfactant ursächlich für die pulmonale bakterielle Translokation sein kann.

Neuere Studien konnten demonstrieren, daß hohe Tidalvolumina nicht nur zu einer Überdehnung der Alveole führen, sondern auch die Freisetzung von proinflammatorischen Mediatoren stimulieren kann [90, 122, 125]. Die Druck-Volumen-Beziehungen sowie verschiedene Zytokine sind in der isolierten Rattenlunge während Beatmung mit hohen Tidalvolumina und hohem PEEP sowie während hoher Tidalvolumina und ZEEP im Vergleich zu einer Kontrollgruppe von der Gruppe um Slutsky [122] untersucht worden. Maschinelle Beatmung mit hohen Tidalvolumina und hohem PEEP führte in dieser Studie zu keiner Änderung der Druck-Volumen-Kurve, während es in der Gruppe mit hohen Tidalvolumina und ZEEP zu einem signifikanten Anstieg von TNF $\alpha$, IL-1 $\beta$, IL-6, INF $\gamma$ und IL-10 in der BAL kam. Diese Zunahme der proinflammatorischen Mediatoren war vergesellschaftet mit einer Abnahme der Compliance und einer Rechts-Verschiebung der Druck-Volumen-Kurve. Der Nachweis erhöhter Aktivität von Kollagenasen (Metalloproteinasen) in der BAL (mittels Gelatinolyse-Aktivität und SDS-PAGE-Zymographie) und einer erhöhten alveolären und interstitiellen mRNA-Expression dieser Enzyme in der Rattenlunge nach maschineller Beatmung für nur 20 Minuten ist kürzlich von Pardo und

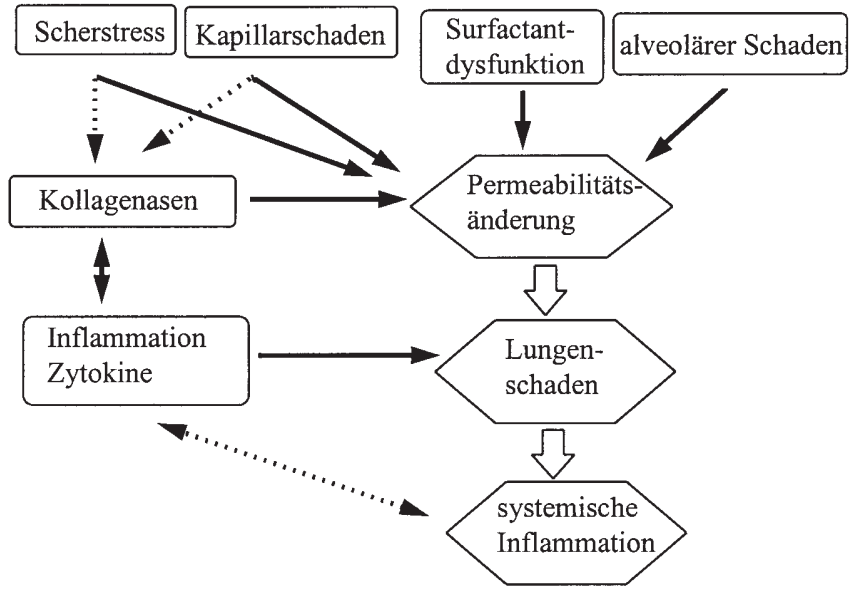

Abb. 1 Schematische Darstellung der „Mechanotransduktion“, einer Sequenz von Ereignissen, die über einen durch Beatmung induzierten, mechanischen Streß zu Permeabilitätsänderungen, Lungenschaden und systemischer Inflammation führt, nach: Sznajder et al. [121].

Mitarbeiter berichtet worden [90]. Eine erhöhte Aktivität von Kollagenasen ist darüber hinaus als Mechanismus der Permeablitätveränderungen unter alveolärer Hyperoxie identifiziert worden [90]. In anderen, kürzlich publizierten Studien ist ein Zusammenhang zwischen mechanischem Stress der Lunge und der Freisetzung von reaktiven Sauerstoffspezies und Induktion von Lipidperoxidation postuliert worden [55, 132]. Neben anderen schlagen Sznajder und Mitarbeiter [119] aufgrund dieser neueren, noch vorläufigen Befunde ein erweitertes Konzept der $\mathrm{Ge}$ nese von beatmungsinduzierten Lungenschäden vor, in dem über eine „Mechanotransduktion“ die Produktion von $\mathrm{O}_{2}$-Radikalen und die Freisetzung von proinflammatorischen Zytokinen stimuliert wird. Dies führt zur Zunahme der Permeabilität und triggert eine Kaskade, an deren Ende die Aggravierung des Lungenschadens und eine systemische Inflammation stehen kann [119]. Das Konzept der Mechanotransduktion ist komplementär zu den „,klassischen" Ursachen des beatmungsinduzierten Lungenschadens. Als solche gilt die Beatmung mit hohen Tidalvolumina, die zu einem direkten mechanischen Trauma mit Bersten des alveolären Epithels, der Zunahme von Scherkräften in den distalen Atemwegen und den Alveolen, Dehnungstrauma des Interstitiums der Kapillaren und Surfactantdepletion führt [28, 29].

Eine maschinelle Beatmung mit PEEP führte schon in frühen tierexperimentellen Untersuchungen zu einer Reduktion des Lungenödems im Vergleich zu Kontrolltieren, die ohne PEEP beatmet wurden [133]. Der pulmonale Gasaustausch der mit PEEP beatmeten Tiere war verbessert [132] und das Alveolarepithel geringer geschädigt [21]. Protektive Effekte von PEEP sind verschiedentlich bei Ratten beschrieben und mit einer besser erhaltenen SurfactantAktivität erklärt worden. 
Tab. 1 Protektive Effekte von PEEP

\begin{tabular}{|c|c|c|}
\hline Verbesserte Oxygenierung & Verringertes Pneumonierisiko & $\begin{array}{l}\text { Verminderung von } \\
\text { Scherkräften }\end{array}$ \\
\hline $\begin{array}{l}\text { - Verbesserung des } \\
\text { Ventilations/Perfusions- } \\
\text { verhältnisses } \\
\text { - Reduktion des intra- } \\
\text { pulmonalen Shunts } \\
\text { - Verbesserte Gasverteilung } \\
\text { endinspiratorisch }\end{array}$ & $\begin{array}{l}\text { - Belüftung und Offenhalten } \\
\text { der basalen Lungen- } \\
\text { abschnitte } \\
\text { - Verminderte Atelektasen- } \\
\text { bildung }\end{array}$ & $\begin{array}{l}\text { - Verhinderung des alveolären } \\
\text { De-Rekruitment } \\
\text { - Verhinderung des zyklischen } \\
\text { Kollaps und Wiedereröffnung } \\
\text { von Alveolen } \\
\text { - Reduktion des intraalveolären } \\
\text { Ödems, Umverteilung des } \\
\text { interstitiellen Ödems }\end{array}$ \\
\hline
\end{tabular}

Dreyfuss und Mitarbeiter [27] zeigten hingegen, daß eine PEEP-induzierte Verringerung der pulmonalen Durchblutung die Entwicklung eines Lungenödems verlangsamte. Wurde Dopamin zur Stabilisierung der Hämodynamik eingesetzt, war das Ausmaß des Lungenödems in der PEEP-Gruppe ebenso ausgeprägt wie in der ZEEPGruppe [27]. Ebensowenig konnte in einer älteren klinischen Studie die Entwicklung eines ARDS durch die prophylaktische Applikation von PEEP vermieden werden [94].

\section{Druck- und volumenlimitierte Beatmung beim ARDS}

Inwieweit ein bestimmtes Beatmungsverfahren mit einer geringeren Schädigung der Lunge assoziiert ist oder zu einer verbesserten Überlebensrate bei ARDS Patienten führt, ist derzeit nicht eindeutig geklärt. Wohl aber haben sich Parameter der maschinellen Beatmung herauskristallisiert, deren Implementation in die Beatmungstherapie Grundlage einer ,lungenprotektiven Beatmungsstrategie“ " [2] geworden sind. Dazu gehören:

- die Anwendung von PEEP

- die Begrenzung der Atemwegsspitzendrucke auf maximal $30-35 \mathrm{~cm} \mathrm{H}_{2} \mathrm{O}$ und der Atemzugvolumina auf etwa 4-8 $\mathrm{ml} / \mathrm{kg} \mathrm{KG}$

- die Beatmung im Bereich der optimalen Compliance des gesamten respiratorischen Systems. [6, 9, 112]

\section{PEEP}

Die Anwendung von PEEP ist seit Jahrzehnten ein integraler Bestandteil in der Beatmungstherapie des ARDS. Schon bei der Erstbeschreibung des „Acute Respiratory Distress in Adults" von David G. Ashbaugh und D. Boyd Bigelow [7] wurde die Applikation von PEEP als sehr ,hilfreich bei der Bekämpfung von Atelektasen und Hypoxie" beschrieben. Neben anderen [58, 75, 76, 108] konnten Kumar und Falke bereits in den 70'Jahren [31, 63] zei- gen, daß es unter der Beatmung mit PEEP zu einer deutlichen Verbesserung der Oxygenierung und Reduktion des intrapulmonalen Shunts kam, ohne daß eine nennenswerte hämodynamische Beeinträchtigung beobachtet wurde. Ebenfalls konnten diese Autoren [64] nachweisen, daß bei Patienten mit ARDS während Beatmung mit PEEP die Inzidenz eines Barotraumas nicht erhöht war. In den folgenden Jahren versuchten verschiedene Arbeitsgruppen an Hand von Veränderung unterschiedlicher physiologischer Parameter unter Beatmung eine Methode zur Einstellung des optimalen PEEP zu definieren.

So beschrieben Kirby et al. [58] bei Patienten mit akutem Lungenversagen die signifikante Abnahme des intrapulmonalen Shunts und Murray et al. [79] im Tierexperiment den Gradienten zwischen $\mathrm{PaCO}_{2}$ (arterielle $\mathrm{CO}_{2}$ Konzentration) und $\mathrm{PetCO}_{2}$ (exspiratorisches $\mathrm{CO}_{2}$ ) als sensitiven Indikator zur Bestimmung des „optimalen PEEP“. Der $\mathrm{PaCO}_{2}-\mathrm{PetCO}_{2}$ Gradient war bei maximalem Rekruitment durch PEEP am kleinsten. Im Unterschied zu Größe des intrapulmonalen Shunt und des $\mathrm{O}_{2}$-Partialdrucks, die unverändert blieben, stieg mit Zunahme der PEEP-induzierten Hyperinflation der $\mathrm{PaCO}_{2}-\mathrm{PetCO}_{2}$ Gradient, als Ausdruck einer zunehmenden Totraumventilation, signifikant an. Suter et al. fanden, daß der PEEPWert, bei dem es zu einer maximalen Steigerung des $\mathrm{O}_{2-}$ Transport kam, einherging mit einer größtmöglichen $\mathrm{Zu}$ nahme der statischen Compliance und bezeichnete diesen PEEP-Wert als „best PEEP“ [115]. Parallel zur Zunahme der Compliance fanden die Autoren eine Abnahme der Totraumventilation.

Die lungenprotektiven Effekte von PEEP (s. Tab. 1) bei der Beatmung von Patienten im akuten Lungenversagen, bestehen in der Verhinderung eines alveolären De-Rekruitments. Dies führt, wie Gattinoni und Mitarbeiter [40] anhand eindrucksvoller CT-Untersuchungen zeigten, zu einer Zunahme der funktionellen Residualkapazität (FRC) sowie zu einer verbesserten inspiratorischen Gasverteilung. Damit verbesserte sich das $\dot{\mathrm{V}}_{\mathrm{A}} / \mathrm{Q}$-Verhältnis, es kam zu einer Reduktion des intrapulmonalen Shunts und letztlich zu einer verbesserten Oxygenierung. In einer weiteren Untersuchung der Gruppe um Gattinoni wurde deutlich [93], daß beim ARDS die Konsolidierungen in den po- 
Abb. 2 Darstellung der Druck-/Volumenbeziehung einer Patientin im akuten Lungenversagen (39 Jahre, ARDS infolge Blut-Aspiration und

hämorrhagischem Schock). Aufzeichnung 3 Tage nach stationärer Aufnahme mit Hilfe der Okklusionstechnik der Evita II Version 76; RV = Residualvolumen, $\mathrm{VT}=$ Atemzugvolumen, TLK = totale Lungenkapazität, LIP = ,lower inflection point", UIP = ,upper inflection point"

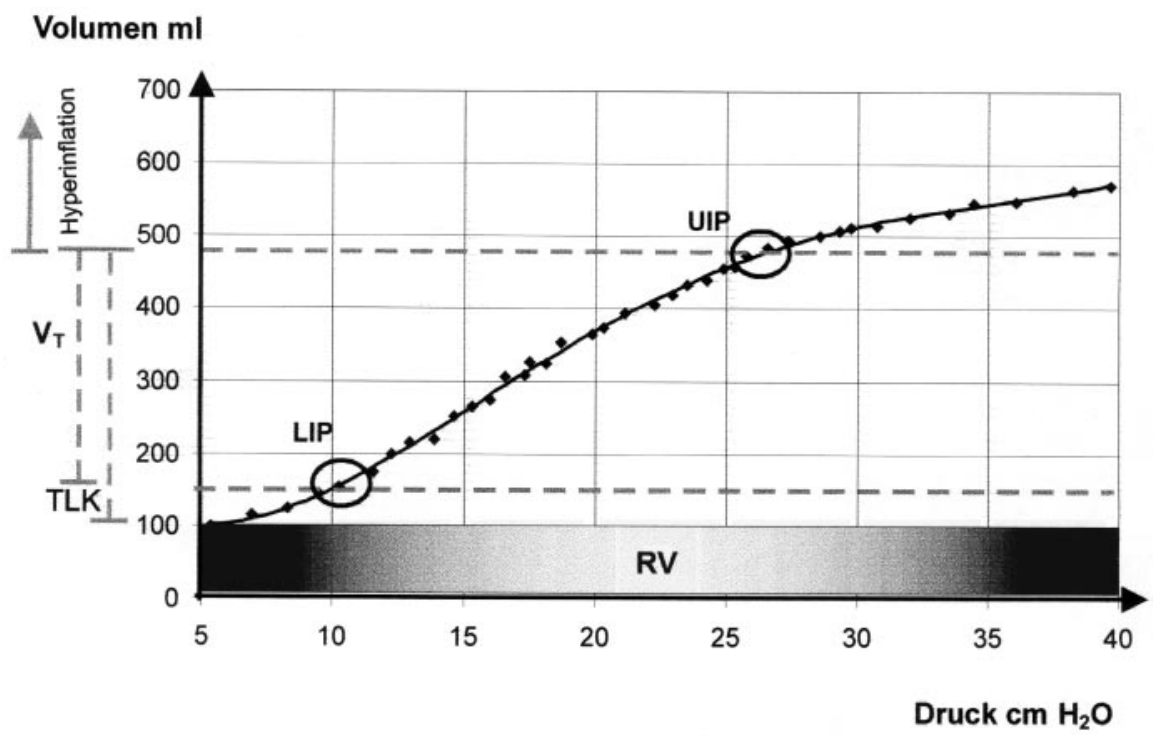

sterioren, abhängigen Lungenpartien entlang eines ventrodorsalen Gradienten zunehmen und parallel dazu der Gas/Gewebequotient exponentiell abnimmt.

Die PEEP-induzierte Rekrutierung alveolärer Gasräume nahm in einer Arbeit von Gattinoni et al. [38] mit ansteigendem PEEP in den entscheidenden, posterioren Lungenpartien anteilmäßig mehr zu, als in den mittleren bzw. ventralen, weniger konsolidierten Abschnitten. Es kam zu einer homogeneren Verteilung des pulmonalen Gasvolumens. Ein hoher PEEP führte zu einer signifikanten $\mathrm{Ab}-$ nahme von Lungengewebe, welches zyklisch kollabierte und wiedereröffnet werden mußte. Die Verminderung der während der zyklischen Wiedereröffnung mechanisch wirksamen Kräfte scheint eine wesentliche Ursache für die Reduktion eines beatmungsassoziierten Lungenschadens $\mathrm{zu}$ sein. Pathophysiologisch spielen neben einer verringerten Auswaschung von Surfactant [66] eine verminderte Schädigung der alveokapillären Basalmembran [13, 26, 80, 91] sowie eine verminderte Ausschüttung proinflammatorischer Zytokine eine Rolle [121, 122, 127], die ihrerseits eine Schädigung der Lunge aggravieren können.

Wie Messung der Druck-/Volumenbeziehung [17, 92, 99] (s. Abb. 2) sowie CT-Untersuchungen gezeigt haben, ist der ,ideale PEEP“ eine individuelle Größe, die ganz wesentlich von der zugrundeliegenden Erkrankung bestimmt wird. In einer neueren Arbeit der Gruppe um Gattinoni [39] wurde gezeigt, daß das Rekruitment alveolärer Gasaustauschfläche durch PEEP bei Patienten mit primär pulmonalem ARDS (Pneumonie) signifikant geringer war als bei Patienten mit primär extrapulmonalem ARDS (Polytrauma, Peritonitis).

Damit ist die Einstellung eines an die Situation des Patienten angepaßten PEEP sowie die Begrenzung des inspiratorischen Druckniveaus und der inspiratorischen
Atemzugvolumina eine in der Praxis wichtige, aber nicht immer einfache Maßnahme, die dazu beiträgt, den beatmungsinduzierten Lungenschaden zu minimieren $[25,26$, $27,35]$.

\section{Die Bestimmung von Druck-Volumen-Beziehungen in der Beatmungstherapie}

Durch bettseitige Messung der statischen Druck/Volumenkurve [98] lassen sich individuelle Druck/Volumenverhältnisse bestimmen, bei denen einerseits ein De-Rekruitment (Unterer Umschlagspunkt, Lower Inflection Point, LIP) sowie andererseits eine Überdehnung der Lunge verhindert wird (Oberer Umschlagspunkt, Upper Inflection Point, UIP). Damit ließe sich idealerweise die Beatmung so an die Situation des Patienten anpassen, daß mit Hilfe der Applikation eines geeigneten PEEP einerseits sowie der Begrenzung des oberen inspiratorischen Druckniveaus (druckkontrolliert) bzw. des inspiratorischen Atemzugvolumens (volumenkontrolliert) andererseits, eine Beatmung im Bereich optimaler Druck/Volumenbeziehung (Abb. 2) des respiratorischen Systems stattfindet $[41,116]$.

Roupie und Mitarbeiter [104], die 25 Patienten mit akutem Lungenversagen und eine Kontrollgruppe von 17 lungengesunden, beatmeten Patienten untersuchten, konnten zeigen, daß bei Patienten mit ARDS die Beatmung mit einem Atemzugvolumen von $10 \mathrm{ml} / \mathrm{kg}$ zu inspiratorischen Plateaudrucken führte, die im Mittel oberhalb des UIP lagen. In der Kontrollgruppe ließ sich bis zu einem inspiratorischen Atemzugvolumen von $1600 \mathrm{ml}$ und einem korrespondierenden inspiratorischen Spitzendruck von $50 \mathrm{~cm}$ 
$\mathrm{H}_{2} \mathrm{O}$ kein UIP nachweisen. Im Mittel lag der obere Umschlagspunkt (UIP) bei den ARDS Patienten bei $26 \pm 6$ $\mathrm{cmH}_{2} \mathrm{O}$, und das korrespondierende Atemzugvolumen, bei welchem der UIP nicht überschritten wurde, betrug im Mittel $7,8 \mathrm{ml} / \mathrm{kg}$. Bei Untersuchungen zur Lungenmechanik von Patienten mit ARDS in Abhängigkeit vom PEEP-Niveaus [17] und der bereits zitierten Untersuchung von Gattinoni et al. [38, 41] konnte einerseits eine Zunahme des alveolären Rekruitment in Abhängigkeit vom PEEP-Niveau gezeigt werden. Andererseits wurde deutlich [41], daß die lungenmechanischen Eigenschaften der kranken, nicht rekrutierbaren Lungenareale in der Druck-/Volumenkurve nicht erfaßt werden. Die in der Druck-/Volumenkurve gemessene Compliance reflektiert hingegen die lungenmechanischen Eigenschaften bzw. Veränderungen der verbliebenen gesunden und auch der rekrutierbaren Lungenareale, die für sich betrachtet annähernd normale lungenmechanische Eigenschaften aufweisen. So läßt sich, dieser Studie zufolge, mit Hilfe der Druck-/Volumenkurve nur indirekt das Ausmaß der inhomogenen Verteilung atelektatischen und gesunden Bezirken in der Lunge abschätzen. Dambrosio et al. [23] zeigten in computertomographischen Untersuchungen, daß die Beatmung mit PEEP zu einer signifikanten Abnahme hyperdenser Lungenareale führt, es aber auch zu einer signifikanten Hyperinflation gesunder Lungenareale kommt. Weiterhin konnte diese Untersuchung zeigen, daß eine Erhöhung der inspiratorischen Atemwegsdrücke oberhalb des UIP keine zusätzlichen Rekrutierung von alveolärer Gasaustauschfläche erbringt, sondern im Wesentlichen zu einer Überdehnung von bereits rekrutierten Arealen führt.

\section{Welches ist das geeignete Beatmungsverfahren für die Therapie des ARDS?}

Aus dem oben Gesagten wird deutlich, daß eine rationale Beatmungstrategie darauf abzielen muß, rekrutierbare Lungenareale zu eröffnen und offenzuhalten, ohne dabei bereits eröffnete Bezirke zu überdehnen (Hyperinflation) und damit deren morphologische Integrität zu zerstören [71].

Diese Vorstellungen lassen sich mit verschiedenen Beatmungsmodi erreichen. Vorteile, die eine druckkontrollierte Beatmung gegenüber einer volumenkontrollierten Beatmung haben könnte, ergeben sich aus Unterschieden im Druck- und Flußverhalten [72].

Bei der druckkontrollierten Beatmung werden infolge des initial hohen inspiratorischen Spitzenflusses potentiell mehr Alveolen rekrutiert als bei der volumenkontrollierten Beatmung. Aufgrund des über die gesamte Inspirationszeit aufrechterhaltenen Druckplateaus (,early and sustained pressure"), welches zu einem dezelerierenden inspiratorischen Fluß führt, kommt es zu einer gleichmäßigeren Umverteilung von Gas in Lungenkompartimente mit langsamen Zeitkonstanten. Unklar ist, ob durch den initial hohen inspiratorischen Spitzenfluß vergleichbar größere Scherkräfte in der Eröffnungsphase der Alveolen auftreten [72].

Das über die gesamte Inspirationszeit aufrechterhaltene Druckplateau begünstigt möglicherweise das Rekruitment kollabierter Lungenareale. Der limitierte inspiratorische Spitzendruck verhindert die dynamische Überblähung von Lungenarealen mit schneller Zeitkonstante (relativ gesunde Lungenareale) und führt damit zu einem verbesserten Ventilations-/Perfusionsverhältnis. Gleichzeitig wird über die Reduktion des inspiratorischen Spitzendruckes die druckinduzierte Schädigung der alveolokapillären Membran vermindert.

In den Tabellen $2 \mathrm{a}-\mathrm{c}$ sind neuere Studien $[2,3,11,67$, $100,114,117]$ aufgeführt, die kontrolliert, prospektiv randomisiert druckkontrollierte und volumenkontrollierte Beatmung im Hinblick auf Parameter des Gasaustausches und der Atemmechanik bei der Behandlung von Patienten mit akutem Lungenversagen untersucht haben. In zwei der Studien $[2,114]$ wurden darüber hinaus auch die Auswirkung der verschiedenen Beatmungsmodi auf Beatmungsdauer und Überlebensrate untersucht.

Wie aus Tabelle $2 \mathrm{a}, \mathrm{b}$ ersichtlich wird, lassen sich die Daten aufgrund der unterschiedlichen Studienprotokolle $[2,3,11,67,100,114,117]$ nicht direkt miteinander vergleichen. Dennoch können die Ergebnisse nebeneinander gestellt und diskutiert werden.

Allen Studien gemeinsam ist, daß es unter der Beatmung im druckkontrollierten Modus zu einer signifikanten Reduktion der inspiratorischen Spitzendrücke sowie zu einer Reduktion der Atemzugvolumina kam. Die Verschlechterung der Oxygenierung während druckkontrollierter Beatmung im umgekehrten Atemzeitverhältnis, I:E $=2: 1$, (Pressure Controlled Inverse Ratio Ventilation, pcIRV 2:1) [67] führen die Autoren auf eine Einschränkung der kardiovaskulären Funktion unter den erhöhten Atemwegsmitteldrücken zurück.

Übereinstimmend fanden hingegen Amato und Mitarbeiter [2, 3] sowie Sydow und Mitarbeiter [117] eine Verbesserung des pulmonalen Gasaustausches, eine Abnahme der Atemwegsmitteldrucke sowie eine Zunahme der Compliance. Erklärt werden diese Veränderungen mit einem zunehmendem Rekruitment alveolärer Gasaustauschfläche und einer Abnahme des intrapulmonalen Shunt bedingt durch eine druckkontrollierte Beatmung mit PEEP. Sydow und Mitarbeiter führten in ihrer Arbeit noch den zunehmenden Anteil an Spontanatmung der Patienten, die in einem modifizierten p-CMV-Modus, dem APRV-Modus (airway pressure release ventilation), beatmet wurden, als Ursache für ein verbessertes Rekruitment der Lunge an. Bemerkenswert ist auch, daß es trotz einer Erhöhung des PEEP-Niveaus in beiden Arbeiten nicht zu einer Erhöhung der inspiratorischen Spitzendrücke kam, was die verbesserte Compliance der Lunge belegt. In keiner der Studien wird von einer erhöhten Pneumothoraxrate unter der druckkontrollierten Beatmung mit hohen PEEP-Niveaus berichtet. Vielmehr kam es in der Arbeit von Amato et al. 
Tab. 2 a, b Zusammenstellung randomisiert, prospektiver Studien (1994-1998) [2, 3, 11, 67, 100, 114, 117], die den Einfluß druckkontrollierter gegenüber volumenkontrollierter Beatmung (p-CMV versus v-CMV) auf den Gasaustausch, atemmechanische Größen und Überleben der Patienten untersucht haben

a Patientendaten bei Studieneinschluß

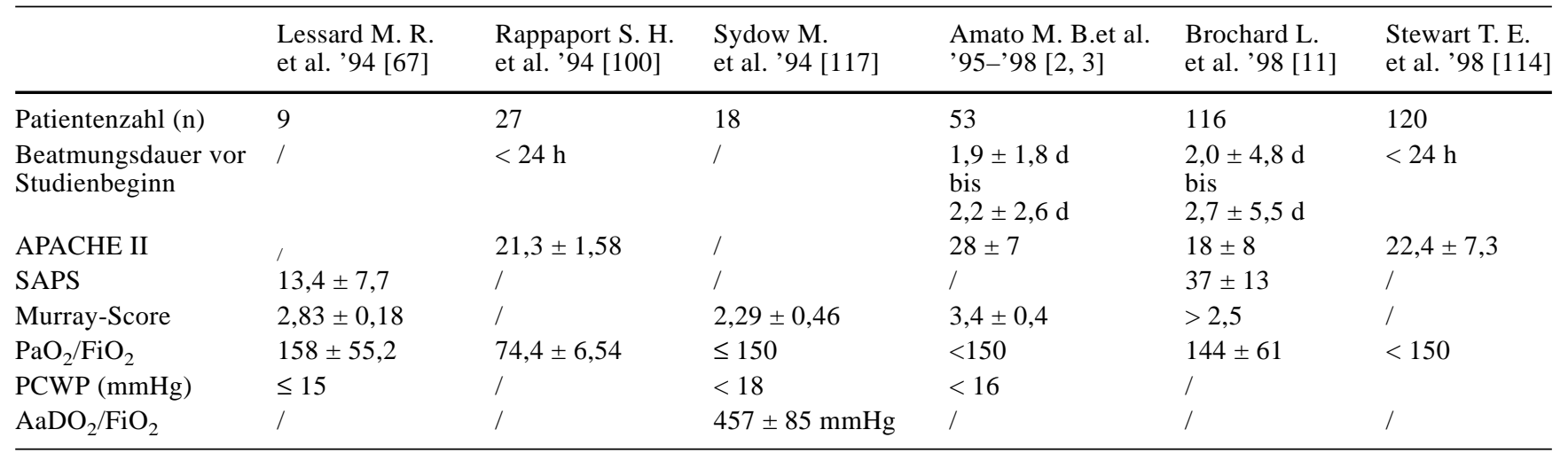

b Studienprotokolle

\begin{tabular}{|c|c|c|c|c|c|c|}
\hline & $\begin{array}{l}\text { Lessard M. R. } \\
\text { et al. '94 [67] }\end{array}$ & $\begin{array}{l}\text { Rappaport S. H. } \\
\text { et al.' } 94 \text { [100] }\end{array}$ & $\begin{array}{l}\text { Sydow M. } \\
\text { et al. '94 [117] }\end{array}$ & $\begin{array}{l}\text { Amato M. B.et al. } \\
\text { '95-'98 }[2,3]\end{array}$ & $\begin{array}{l}\text { Brochard L. } \\
\text { et al. '98 [11] }\end{array}$ & $\begin{array}{l}\text { Stewart T. E. } \\
\text { et al. '98 [114] }\end{array}$ \\
\hline Studienablauf & $\begin{array}{l}\text { Patienten nach- } \\
\text { einander in } \\
\text { verschiedenen } \\
\text { Modi beatmet }\end{array}$ & $\begin{array}{l}\text { Patienten in } \\
2 \text { Gruppen } \\
\text { randomisiert }\end{array}$ & $\begin{array}{l}\text { Patienten in } \\
2 \text { Gruppen } \\
\text { randomisiert }\end{array}$ & $\begin{array}{l}\text { Patienten in } \\
2 \text { Gruppen } \\
\text { randomisiert }\end{array}$ & $\begin{array}{l}\text { Patienten in } \\
2 \text { Gruppen } \\
\text { randomisiert }\end{array}$ & $\begin{array}{l}\text { Patienten in } \\
2 \text { Gruppen } \\
\text { randomisiert }\end{array}$ \\
\hline $\begin{array}{l}\text { Beatmungs- } \\
\text { modus }\end{array}$ & $\begin{array}{lr}\mathrm{v}-\mathrm{CMV} & \mathrm{I}: \mathrm{E}=1: 2 \\
\text { p-CmV } & 1: 2 \\
\text { pc-IRV } & 2: 1,3: 1\end{array}$ & $\begin{array}{l}\text { v-CMV = konst. } \\
\text { Fluß, ansteigen- } \\
\text { der Druck } \\
\text { p-CMV = dez. } \\
\text { Flu } \beta, \text { konst. } \\
\text { insp. Druck }\end{array}$ & $\begin{array}{l}\text { vc-IRV: konst. } \\
\text { Fluß } \\
\text { APRV = airway } \\
\text { pressure release } \\
\text { ventilation }\end{array}$ & $\begin{array}{l}\text { v-CMV: konst. } \\
\text { Fluß } \\
\text { pc-IRV, PSV }\end{array}$ & $\begin{array}{l}\text { v-CMV (assist- } \\
\text { control) } \\
\text { p-CMV (assist- } \\
\text { control) }\end{array}$ & $\begin{array}{l}\text { v-CMV mit } \\
\text { dez. Fluß } \\
\text { p-CMV }\end{array}$ \\
\hline $\begin{array}{l}\text { PEEP } \\
\left(\mathrm{cm} \mathrm{H}_{2} \mathrm{O}\right)\end{array}$ & 12 & & $\begin{array}{l}5 \text { bei v-CMV } \\
\text { PEEP }_{\text {I }} \text { ? bei APRV }\end{array}$ & $\begin{array}{l}16 \text { Reduktion auf } \\
13 \text { nach } 36 \text { Std. }\end{array}$ & $10 \pm 2$ & $9 \pm 4$ \\
\hline $\begin{array}{l}\text { Spontan- } \\
\text { atmung }\end{array}$ & nein & & $\begin{array}{l}\text { bis } 30 / \mathrm{min} \\
\text { bei APRV }\end{array}$ & ja & & $\begin{array}{l}\text { Relaxiert in p-CMV } \\
\text { Gruppe }\end{array}$ \\
\hline
\end{tabular}

APACHE II = Acute Physiology And Chronic Health Evaluation II, SAPS = Simplified Acute Physiology Score,

$\mathrm{FiO}_{2}=$ fraction of inspired oxygen, $\mathrm{PaO}_{2}=$ Sauerstoffpartialdruck, , PCWP $=$ Pulmonary Capillary Wedge Pressure, AaDO $2=$ Alveolararterielle $\mathrm{O}_{2}$-Partialdruck-Differenz, PEEP = Positive End-Expiratory Pressure

[2] zu einer signifikanten Abnahme von Barotraumata in der druckkontrolliert beatmeten Patientengruppe. Diese Befunde sind einerseits gut vereinbar mit einer geringeren Wirkung von Scherkräften in der einmal eröffneten und dann offengehaltenen Lunge, andererseits sprechen sie in Übereinstimmung mit oben zitierten tierexperimentellen Arbeiten [26] für eine wirksame Lungenprotektion durch eine Reduktion der inspiratorischen Spitzendrucke. 
Tab. 2 c Ergebnisse der Studien [2, 3, 11, 67, 100, 114, 117], die den Einfluß druckkontrollierter gegenüber volumenkontrollierter Beatmung auf den Gasaustausch, atemmechanische Größen und Überleben der Patienten wiedergeben

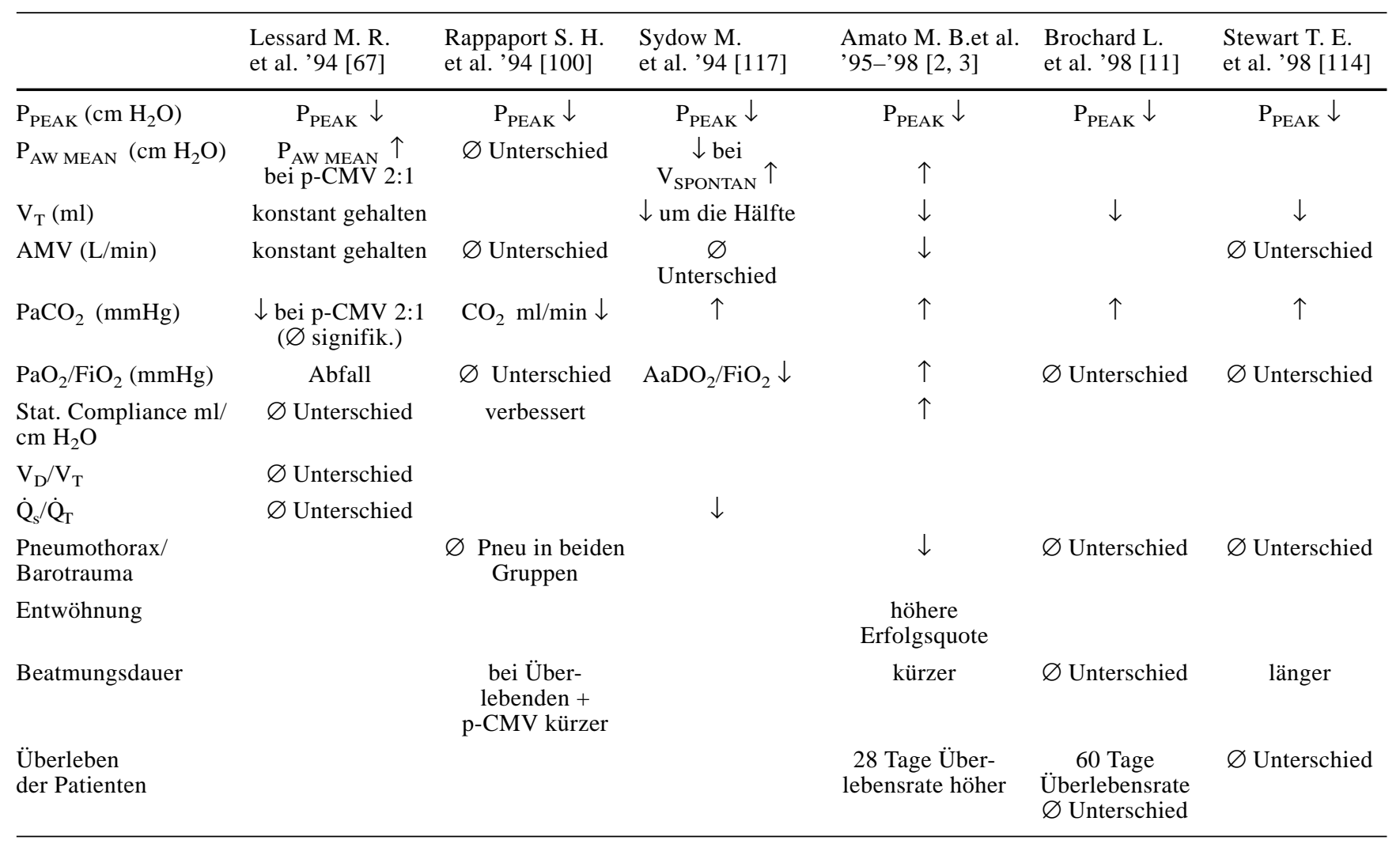

$\mathrm{PaCO}_{2}=$ Kohlendioxidpartialdruck, $\mathrm{PaO}_{2}=$ Sauerstoffpartialdruck, stat. Compliance = statische Compliance, $\mathrm{V}_{\mathrm{D}}=$ Totraumventilation, $\mathrm{V}_{\mathrm{T}}=$ totale Ventilation, $\dot{Q}_{S} / \dot{Q}_{T}=$ intrapulmonaler Shunt, $\mathrm{P}_{\mathrm{PEAK}}=$ inspiratorischer Spitzendruck, $\mathrm{P}_{\mathrm{AW} \text { MEAN }}=$ mittlerer Atemwegsdruck, $\mathrm{V}_{\mathrm{T}}=$ Atemzugvolumen, AMV $=$ Atemminutenvolumen

Es bleibt Hypothese, ob die von Sydow et al. und Amato et al. gefundene Zunahme der statischen Compliance und die verbesserte Oxygenierung einen Heilungsprozess von geschädigten Lungenarealen während druckkontrollierter Beatmung mit PEEP reflektiert. Dafür spricht eine von Amato und Mitarbeiter gefundene Zunahme der ,beatmungsfreien Tage" und der Überlebensrate nach 28 Tagen in der druckkontrolliert beatmeten Patientengruppe.

Den Ergebnissen dieser Arbeiten stehen die Untersuchungen von Brochard et al. [11] und Stewart et al. [114] entgegen. Stewart und Mitarbeiter untersuchten 120 Patienten im Frühstadium des ARDS bzw. Patienten, die die Kriterien des „Acute lung injury“ (ALI) erfüllten. Sie wurden randomisiert einer Kontrollgruppe (CMV-Beatmung, erlaubter inspiratorischer Spitzendruck $50 \mathrm{cmH}_{2} \mathrm{O}$, $\left.\mathrm{V}_{\mathrm{T}} 10-15 \mathrm{ml} / \mathrm{kg} \mathrm{KG}\right)$ bzw. einer ,limited-ventilation group" (druck- oder volumenlimitierte Beatmung, inspiratorischer Spitzendruck $\leq 30 \mathrm{cmH}_{2} \mathrm{O}, \mathrm{V}_{\mathrm{T}} \leq 8 \mathrm{ml} / \mathrm{kg} \mathrm{KG}$ ) zugewiesen. Der Untersuchungszeitraum betrug 7 Tage. Der PEEP wurde in einem Bereich von $5-20 \mathrm{cmH}_{2} \mathrm{O}$ so eingestellt, daß die $\mathrm{FiO}_{2}$ von 0,5 nicht überschritten wurde. Der applizierte PEEP lag im Mittel bei $8 \mathrm{cmH}_{2} \mathrm{O}$. Es kam zu ei- ner signifikanten Reduktion der Beatmungsdrücke und der Tidalvolumina sowie einer in Ausmaß und Dauer signifikanten Zunahme der Hyperkapnie und einer Erhöhung der Atemfrequenz in der „limited-ventilation group“.

Die Krankenhausüberlebensrate unterschied sich nicht. Vielmehr wurden die Patienten der „limited-ventilation group" länger beatmet, hatten einen längeren Aufenthalt auf der Intensivstation und mußten häufiger dialysiert werden. Allerdings starben in der Kontrollgruppe $n=3$ Patienten, während in der „limited-ventilation group“ alle $\mathrm{Pa}$ tienten das Lungenversagen überlebten.

Die Autoren führen die fehlenden Unterschiede zwischen den beiden Gruppen darauf zurück, daß die Plateaudrücke der Patienten in der Kontrollgruppe, obwohl sie mit inspiratorischen Tidalvolumina von $10-15 \mathrm{ml} / \mathrm{kg} \mathrm{KG}$ beatmet wurden $35 \mathrm{cmH}_{2} \mathrm{O}$ nicht überschritten und die $\mathrm{Pa}$ tienten damit wahrscheinlich in einem Druckbereich beatmet wurden, der unterhalb des oberen Umschlagpunktes und damit im ,sicheren“ Teil der Druck-Volumenkurve lag. Zum anderen vermag diese Studie keine Aussage darüber zu treffen, ob eine druck- und volumenlimitierte Beatmungsstrategie bei Patienten im fortgeschrittenen Stadium 
eines ARDS eine positive Auswirkung auf das Überleben der Patienten gehabt hätte. Die in die Studie eingeschlossenen Patienten befanden sich im Anfangsstadium der Erkrankung, was auch die gute Compliance der Lunge belegt.

Brochard et al. [11], der eine multizentrische Studie durchführte, kommt zu ähnlichen Ergebnissen wie Stewart. Die Patienten, die volumenkontrolliert mit Tidalvolumina von $10 \mathrm{ml} / \mathrm{kg} \mathrm{KG}$ beatmet wurden hatten ähnlich niedrige inspiratorische Plataeudrücke $\left(\sim 30 \mathrm{cmH}_{2} \mathrm{O}\right)$ wie die volumenkontrolliert beatmeten Patienten in der Untersuchung von Stewart. Die 60 Tage Überlebensrate in den beiden Gruppen unterschied sich in Bezug auf die Beatmungsstrategie nicht. Eine signifikant schlechtere Überlebenswahrscheinlichkeit hatten die Patienten, die im Verlauf des ARDS ein sekundäres Mehrorganversagen entwickelten. Die Inzidenz ein Mehrorganversagen zu entwickeln, war in beiden Gruppen mit 24\% identisch.

Die heterogene Ausprägung des ARDS mit unterschiedlichen, nebeneinander vorkommenden pathophysiologischen und pathomorphologischen Veränderungen und unterschiedlichen atemmechanischen Verhältnissen in der Lunge (,capillary-leakage“ Syndrom bei Sepsis, ausgedehnte Infiltrationen des Lungenparenchyms aufgrund einer Pneumonie, Atelektasenbildung und Kontusion nach Thoraxtrauma, fibrotische Veränderungen des Lungenparenchyms z.b. nach Bleomycintherapie), erschweren die Erhebung vergleichbarer Daten im Rahmen von klinischen Studien erheblich [39].

Präklinische Ergebnisse einer derzeit in Nordamerika durchgeführten, prospektiven, randomisierten multizentrischen Studie [5], in die 850 Patienten mit akutem Lungenversagen eingeschlossen wurden, legen nahe, daß die Beatmung mit niedrigen Atemzugvolumina ( $6 \mathrm{ml} / \mathrm{kg} \mathrm{KG})$ und niedrigen inspiratorischen Plateaudrücken (25 $\mathrm{cmH}_{2} \mathrm{O}$ ) zu einer signifikanten Reduktion der Letalität und der Beatmungsdauer führen kann. Die Studie, ursprünglich auf die Untersuchung von 1000 Patienten angelegt, wurde nach der vierten Interimsanalyse aufgrund der signifikanten Ergebnisse abgebrochen.

Aus den dargestellten Studien kann geschlußfolgert werden, daß weniger durch die Anwendung eines bestimmten Beatmungsverfahrens per se, sondern vielmehr durch die Implementierung bestimmter, protektiver Beatmungsstrategien, wie der Applikation eines ausreichenden PEEP, der Begrenzung des oberen inspiratorischen Druckniveaus und der inspiratorischen Atemzugvolumina ein beatmungsassoziierter Lungenschaden vermieden werden kann.

\section{Adjuvante Maßnahmen bei Anwendung einer lungenprotektiven maschinellen Beatmung}

Es werden zur Zeit verschiedene neue Therapieansätze evaluiert, die zu einer Verbesserung des Gasaustausches führen können und deren Rationale eine Reduktion des durch die maschinelle Beatmung induzierten Lungenschadens bei Patienten mit schwerem ARDS ist: Inhalation von Stickstoffmonoxid, intratracheale Surfactantinstillation, partielle Flüssigkeitsbeatmung mit Perfluorcarbonen, High-Frequency-Ventilation und tracheale Gasinsufflation [74]. Daneben haben sich die Anwendung der permissiven Hyperkapnie und die Beatmung in Bauchlage bereits fest in die ARDS-Therapieschemata integriert, da durch diese Maßnahmen mit geringem technischem Aufwand häufig ein großer therapeutischer Fortschritt für den Patienten erzielt werden kann.

\section{Inhalation von Stickstoffmonoxid (NO) und Vernebelung von Prostacyclin}

Pulmonalvaskuläre Obstruktion, Obliteration und Konstriktion führen beim ARDS-Patienten zu der charakteristischen pulmonalarteriellen Hypertension. Durch medikamentöse Aufhebung der hypoxievermittelten Vasokonstriktion, d. h. systemische Gabe von Vasodilatatoren allein würde sich auch ohne Bestehen einer pulmonalen Hypertonie kein normales Ventilations-/Perfusionsverhältnis einstellen, ein vergrößerter intrapulmonaler Rechts-Links-Shunt und eine Verschlechterung der Oxygenation des Patienten wären die Folge [4, 44]. Die pulmonalarterielle Vasokonstriktion kann durch vasoaktive Substanzen wie Nitrate, Nitroprussid, Kalziumkanalantagonisten, Bronchodilatatoren und Inhalationsanästhetika beeinflußt werden. Aufgrund der systemischen Wirkungen dieser Substanzen ist ihre therapeutische Anwendung zur Senkung des pulmonalarteriellen Druckes limitiert [4, 44].

Palmer und Mitarbeiter [88] berichteten, daß im Gefäßendothel Stickstoffmonoxid (NO) gebildet wird, das relaxierende Wirkung auf die glatte Gefäßmuskulatur ausübt. Frostell und Mitarbeiter [34] zeigten im Tierversuch, daß durch die Inhalation von NO eine pulmonalvaskuläre Konstriktion in den ventilierten Lungenarealen aufgehoben werden kann, ohne daß ein Einfluß auf den systemischen vaskulären Widerstand, das Herzzeitvolumen, den PCWP oder den zentralvenösen Druck nachgewiesen werden konnten. Durch die selektive Vasodilatation ventilierter Lungenbezirke mit konsekutiver Verbesserung der Durchblutung kann bei einem Teil der ARDS-Patienten eine deutliche Verbesserung der arteriellen Oxygenation erreicht werden. Rossaint und Mitarbeiter [102] behandelten 9 Patienten mit ARDS mit inhalativem NO in zwei verschiedenen Konzentrationen über jeweils 40 Minuten. Während der Zufuhr von 18 ppm (parts per million) sank der mittlere PAP von 37 auf $30 \mathrm{mmHg}$, der intrapulmonale Shunt reduzierte sich von 36 auf $31 \%$. Der mittlere arterielle Druck und das Herzzeitvolumen blieben unbeeinflußt. Bigatello und Mitarbeiter [10] berichteten später über die Kurz- und Langzeitanwendung von NO bei 13 ARDS-Pa- 
tienten. In dieser Untersuchung führte die Inhalation von 2-40 ppm zu einer Reduktion des mittleren pulmonalarteriellen Drucks und verbesserte die Oxygenation ohne Beeinflussung der systemischen Hämodynamik.

Gerlach und Mitarbeiter [43] konnten anhand von Dosis-Wirkungskurven zeigen, daß bereits sehr niedrige mittlere effektive Dosen (ED50) im Bereich von 100 ppb (parts per billion) zu einer Verbesserung der Oxygenation führen können. Eine Abnahme des mittleren PAP wurde erst bei einer ED50 von 2-3 ppm beobachtet. In einer weiteren Studie konnten Gerlach und Mitarbeiter [42] eine Verbesserung des Oxygenationsindexes um 30\% durch Dosen von 60, 100 und $230 \mathrm{ppb}$ ohne Beeinflussung des mittleren PAP feststellen. Eine Verbesserung der Oxygenation kann also auch ohne Beeinflussung des pulmonalvaskulären Widerstandes eintreten; inhaliertes NO hat mit großer individueller Varianz unterschiedliche Wirkungen in niedrigen bzw. hohen Konzentrationen im Atemgas.

Rossaint und Mitarbeiter [103] untersuchten in einer retrospektiven Studie bei 87 ARDS-Patienten den Einfluß der inhalativen NO-Applikation auf Gasaustausch, Hämodynamik und Überlebensrate. Die Autoren fanden eine deutliche Verbesserung der Oxygenation und eine Verringerung der mittleren pulmonalarteriellen Drücke, jedoch keinen Einfluß auf die Überlebensrate des ARDS. Brett et al. [12] untersuchten 26 Patienten mit ARDS bezüglich der Effektivität von iNO. Vierzehn Patienten wurden als Responder, $\mathrm{n}=12$ als Non-Responder identifiziert. Beide Untersuchungsgruppen zeigten bezüglich CT-Scoring, Inflammationsparametern der bronchoalveolären Lavage (BAL), Oxygenierung, Lung Injury Score, pulmonalvaskulärem Widerstand und Überlebensrate keinen Unterschied.

Mehrere klinische und tierexperimentelle Untersuchungen brachten bezüglich der Wirkung von iNO auf die Inflammation des Lungengewebes und möglicher lungenprotektiver Wirkungen beim ARDS gegensätzliche Ergebnisse. Die Arbeitsgruppe von Payen konnte anhand von Untersuchungen zur Funktion alveolärer neutrophiler Granulozyten und von Zytokinspiegeln in der BAL Hinweise für eine Reduktion der pulmonalen Inflammation unter Inhalation von NO finden [18]. Zhu et al. [134] hatten im Tierexperiment in Kombination mit exogenem Surfactant ähnliche Ergebnisse, während Nishina et al. in einem durch Endotoxin induziertem ARDS-Modell keinen positiven Einfluß von NO auf den Lungenschaden nachweisen konnten [85].

Die inhalative Anwendung von NO bei ARDS-Patienten ermöglicht in bis zu 60\% der Fälle [24], die Oxygenation zu verbessern und hohe pulmonalarterielle Drucke zu senken. Dadurch könnte es möglich sein, das Risiko von Volu-/Barotraumata bei maschineller Beatmung und toxischen Sauerstoffkonzentrationen zu vermindern. Der Nachweis einer Steigerung der Überlebensrate des ARDS durch Anwendung von inhalativem NO steht jedoch auch weiterhin noch aus $[12,24]$.
Die Vernebelung von Prostacyclin ist eine weitere Möglichkeit einer selektiven Vasodilatation in ventilierten Lungenarealen. Prostacyclin scheint bei diesem Applikationsweg nur gering ausgeprägte systemische Wirkungen zu haben, die Effekte auf den Gasaustausch sind dem des iNO vergleichbar [128, 129, 130].

\section{Surfactant-Applikation}

Da Alveoli mit einem kleinen Durchmesser - dem Gesetz von La Place folgend - dazu neigen, ihr enthaltendes Gasvolumen zu Einheiten mit größerem Durchmesser zu verschieben, muß in der gesunden Lunge die Oberflächenspannung der Alveole durch die Surfactantfunktion herabgesetzt und so die Alveolarwand stabilisiert werden. Beim ARDS ist sowohl die Menge als auch die Qualität des noch vorhandenen Surfactants herabgesetzt $[69,107]$. Diese Veränderungen des Surfactantsystems treten bereits sehr früh im Krankheitsverlauf auf und sind auf eine verminderte Synthese sowie auf eine Inaktivierung durch Exsudation von Plasmaproteinen zurückzuführen. Störungen der endogenen Surfactant-Synthese führen zu Atelektasenbildung und aufgrund anderer biologischer Funktionen auch zu einer Verminderung der lokalen immunologischen Abwehr. Exogenes Surfactant ist in der Lage, den inhibitorischen Effekten der beim ARDS exsudierten Plasmaproteine entgegenzuwirken, wenn er in ausreichender Dosis bis in die Alveolen gelangen kann [65]. Bisher wurden mehrere, in Zusammensetzung und Wirksamkeit unterschiedliche Surfactant-Präparationen entwickelt [47, 48]. Man unterscheidet aus tierischem Gewebe gewonnene Produkte, synthetische und jetzt neuerlich auch gentechnisch hergestellte, sogenannte rekombinante Surfactants. Surfactant kann als Aerosol vernebelt oder direkt (auch gezielt bronchoskopisch) instilliert werden [128]. In der Frühphase des ARDS eingesetzt, kann exogener Surfactant günstige Effekte auf Gasaustausch und Krankheitsverlauf erbringen [128], den Stellenwert wie in der Therapie des Lungenversagens unreifer Neugeborener hat die exogene Surfactanttherapie beim adulten Lungenversagen bisher jedoch nicht erreichen können. Tierexperimentelle Arbeiten an ARDS-Modellen mit endotoxininduziertem Lungenversagen $[84,95]$ zeigten nur geringe antiinflammatorische Effekte und Reduktion des histologischen Lungenschadens durch Applikation von exogenem Surfactant.

\section{Partial Liquid Ventilation mit Perfluorcarbonen (PLV)}

Perfluorcarbone (PFC) sind chemische Verbindungen mit ungewöhnlichen physikochemischen Eigenschaften. Sie sind in der Lage, große Mengen Gas - klinisch eingesetzt 
- Sauerstoff und Kohlendioxid zu lösen. Sie sind biologisch weitgehend inert und sind sowohl mit Wasser als auch mit Fett nicht mischbar. Perfluoroctylbromide (Perfluorbron) ist die klinisch bisher am häufigsten eingesetzte Substanz aus dieser Stoffklasse. Auch bei längerer intratrachealer Anwendung wurden bisher keine pathologischen Gewebsreaktionen in der Lunge beobachtet. Die Flüssigkeit hat wassergleiche Viskosität, verdampft rasch und verteilt sich aufgrund ihrer oberflächenspreizenden Eigenschaft homogen auf allen Oberflächen [30]. Die physikalische Dichte von Perfluorbron ist etwa doppelt so hoch wie die von Wasser. Bronchialsekret schwimmt auf der Flüssigkeit, die Röntgendichte der Substanz ist so hoch, das auf der konventionellen Thoraxaufnahme keine Lungenstrukturen mehr abgrenzbar sind.

Zwei Hauptwirkungen machen Perfluorbron für den Einsatz beim ARDS interessant: (1) Die niedrige Oberflächenspannung erzeugt einen surfactantartigen Effekt, eröffnet so Alveoli und hält sie offen und (2) bei Beatmung mit einer inkompressiblen, jedoch zum Gasaustausch fähigen Flüssigkeit können verschlossene Alveolarräume durch den direkt übertragenen Druck eröffnet werden.

Die PLV stellt ein Hybridverfahren aus Flüssigkeitsbeatmung und konventioneller Beatmung dar, bei der Perfluorbron intratracheal instilliert wird, bis sich ein Spiegel im Tubus zeigt. Der Patient wird weiter wie gewöhnlich mit Atemgas ventiliert. Unter der Anwendung der PLV stellt sich ein der Schwerkraft folgender PEEP-artiger Effekt auf die posterior gelegenen Lungenabschnitte ein, eine Reduktion des intrapulmonalen Shunts und eine deutliche Verbesserung des Gasaustausches konnten mehrfach demonstriert werden $[36,53,54,83,123]$. Es wird angenommen, daß dies Folge der Wiedereröffnung bisher verschlossenen Alveolarraumes und der Umverteilung des pulmonalen Blutflusses hin zu den besser ventilierten nicht-abhängigen Lungenarealen ist.

Bedingt durch die physikalische Dichte von Perfluorbron schwimmt Bronchialsekret auf der Flüssigkeit und scheidet sich zusammen mit Zelldetritus aus dem Bronchialsystem an der Oberfläche ab. Aufgrund der Verdampfung des PFC während der Beatmung, kommt es dann zu einer Eintrocknung des Sekrets. Diese Art von intensiver „Reinigung“ kann bis hin zur Verlegung des Beatmungstubus führen. Wie bei der exogenen Surfactanttherapie ist auch die PLV mit PFC vermutlich besonders wirkungsvoll in der Frühphase des ARDS [22]. Dies ist derzeit Gegenstand einer klinischen Phase III-Studie. Die Anwendung von PLV mit PFC reduzieren die Inflammation des Lungengewebes - gemessen an den proinflammatorischen Interleukinen IL-1 und IL-6 [22] - und kann das Fortschreiten eines beatmungsbedingten Lungenschadens verlangsamen [13, 22, 53, 54]. Die günstige Wirkung von PLV auf pulmonalen Gasaustausch und Lungenmechanik läßt sich durch die Kombination mit PEEP [56, 57, 59] noch weiter steigern.

\section{High Frequency Ventilation (HFV)}

Die Hochfrequenzbeatmung ist eine relativ junge Form der maschinellen Beatmung, die mit sehr geringen Änderungen des Alveolardruckes arbeitet, indem minimale Tidalvolumina, häufig kleiner als der anatomische Totraum, mit extrem hohen Frequenzen appliziert werden. Ein Vorteil des Verfahrens gegenüber konventioneller maschineller Beatmung liegt in der Möglichkeit adäquate Atemminutenvolumina bei niedrigeren proximalen Atemwegsdrücken zu applizieren - häufig auch noch dann, wenn eine kontrollierte maschinelle Beatmung (CMV) dazu nicht mehr in der Lage ist. Es gibt drei Haupttypen von HFV:

(1) High Frequency Positive Pressure Ventilation (HFPPV) ist eine Form von HFV, die von modifizierten konventionellen Ventilatoren appliziert werden kann, die mit hohen Beatmungsfrequenzen arbeiten;

(2) High Frequency Jet Ventilation (HFJV) wird mit Geräten durchgeführt, die einen direkt in die Atemwege gerichteten starken Gasstrom (Jet) erzeugen;

(3) High Frequency Oscillatory Ventilation (HFOV) wird mit Ventilatoren durchgeführt, die eine kleine Gasmenge in den Atemwegen vor und zurück bewegen.

HFV erscheint theoretisch überaus gut geeignet, die Grundprinzipien der lungenprotektiven Beatmung - ein maximales Rekruitment von Alveolarraum bei minimalen Tidalvolumina - umzusetzen [33]. Der nach Entwicklung des Verfahrens und Berichten von kasuistischen Erfolgen anfänglich große Enthusiasmus wurde in den letzten Jahren jedoch gedämpft, da Studien aus dem Bereich der Neonatologie und Kinderheilkunde $[19,120]$ widersprüchliche Ergebnisse zur Wirksamkeit erbrachten. Die Anwendung beim Erwachsenen war bisher durch den Mangel an geeigneten Geräten limitiert, die eine effektive Hochfrequenzbeatmung erlaubten [45]. Daher konnte bisher noch nicht gezeigt werden, daß HFV konventionellen Beatmungsformen in der Therapie des ARDS hinsichtlich der Überlebensrate, der beatmungsfreien Tage oder der Häufigkeit von Baro-/Volutraumata überlegen oder auch nur gleichwertig ist. Insbesondere jedoch bei der Beatmung von Patienten mit „Air-Leak" Syndrom [113] hat sich der Einsatz von HFV als effektiv erwiesen.

\section{Tracheale Gas Insufflation (TGI)}

Die tracheale Insufflation von Frischgas mittels Katheter (TGI) stellt eine alternative Technik bei sich rasch entwickelnder Hyperkapnie dar. Eine Bedeutung im Hinblick auf die Minimierung eines beatmungsassoziierten Lungenschadens könnte die TGI insofern haben, als das mit ihrer Hilfe, falls notwendig, eine effiziente Kohlendioxidelimination möglich ist bei erhaltenen niedrigen Tidalvolumina und Beatmungsdrücken. TGI wirkt durch die 
vermehrte Auswaschung von $\mathrm{CO}_{2}$ aus dem anatomischen Totraum. Zwei Typen von TGI werden unterschieden: (1) TGI mit einem kontinuierlichem Gasfluß unabhängig vom Beatmungsgerät - continuous flow TGI (2) TGI im Beatmungszyklus getriggert - phasic flow TGI $[15,81,82]$. Der wirkungsvolle Anteil der TGI ist der additive Gasstrom in der Exspirationsphase. Abhängig von der eingestellten Flowrate der TGI und der vom Beatmungsgerät durchgeführten Ventilation ändert sich die Effektivität des Verfahrens. Niedrige Flußraten wirken mehr durch reine Totraumauswaschung, hohe Flußraten erzeugen intratracheale Turbulenzen im Atemgas, die neben einem PEEP-Effekt auch noch das in den tieferen Bronchien vorhandene Gas mischen, was zur $\mathrm{CO}_{2}$-Elimination beitragen kann. Je kleiner die applizierten Tidalvolumina sind, desto größer ist der relative Anteil der Totraumventilation am gesamten Atemminutenvolumen, um so wirkungsvoller wird der Einsatz von TGI. Mit Hilfe von „reverse-flush“-TGI Kathetern kann durch nach extrakorporal gerichteten Gasstrom der additive alveoläre Druckanstieg der Technik minimiert werden [78].

TGI ist jedoch auch mit einer Reihe von praktischen Problemen behaftet. Die bisher vorgestellten technischen Lösungen sind bezüglich ihrer Überwachbarkeit und Betriebssicherheit nicht ausgereift, die Gefahr der Austrocknung der Schleimhäute mit allen Folgeproblemen ist groß. Die Verringerung des freien Tubusinnendurchmessers erhöht den Atemwegswiderstand. Letztendlich bleiben auch hygienische Probleme durch räumliche Konkurrenz mit dem Absaugkatheter noch ungelöst. Eine klinische Evaluierung des Verfahrens steht weiterhin aus.

\section{Permissive Hyperkapnie (PHC)}

Bei der Beatmung eines Patienten mit schwerem ARDS sind Normalwerte im Blut für den $\mathrm{PaCO}_{2}$ und $\mathrm{pH}$ häufig nur durch die Anwendung großer Atemzugvolumina verbunden mit hohen Beatmungsdrücken zu erreichen, da die ARDS-typischen pulmonalen Veränderungen - gesteigerter inrapulmonaler Shunt, vermehrte Totraumventilation, hypermetabolische $\mathrm{CO}_{2}$-Produktion - ein deutlich gegenüber der Norm erhöhtes Atemminutenvolumen zur $\mathrm{CO}_{2}$-Elimination erforderlich machen. Maschinelle Beatmung mit großen Tidalvolumina und hohen Spitzendrücken kann jedoch, wie dargestellt, selbst eine progressive Lungenschädigung bewirken. Daher werden als lungenprotektive Beatmungstherapie kleine Atemzugvolumina von $4-8 \mathrm{ml} / \mathrm{kg}$ KG mit individuell titriertem PEEP [73], niedrige Atemwegsspitzendrücke $\left(<35 \mathrm{cmH}_{2} \mathrm{O}\right)$ und eine möglichst niedrige inspiratorische Sauerstoffkonzentration $\left(\mathrm{FiO}_{2}\right)$ eingesetzt. Die Begrenzung von Spitzendruck und Tidalvolumen soll die Lunge vor beatmungsinduziertem Schaden schützen, führt allerdings zu einem Anstieg des $\mathrm{PaCO}_{2}$, dessen Tolerieren ,permissive Hyperkapnie“ (PHC) genannt wird
[124]. Wenn sich die Hyperkapnie über Stunden bis Tage entwickelt, kann der Organismus die Ausbildung einer schweren Azidose durch körpereigene Mechanismen z. B. vermehrte renale Resorption von Bikarbonat - kompensieren. Untersuchungsbefunde bei Patienten mit chronischen Lungenerkrankungen haben gezeigt, daß auch ausgeprägte Hyperkapnie gut toleriert wird, wenn sie sich langsam genug entwickeln kann. Ein durch Verminderung der alveolären Ventilation bedingter Abfall des $\mathrm{PaO}_{2}$ kann durch Erhöhung der $\mathrm{FiO}_{2}$ um 10\% ausgeglichen werden [50]. Mehrere Studien konnten zeigen, daß die Anwendung von PHC die Überlebensrate von ARDS-Patienten steigern kann [2, 3, 51, 52, 68, 77].

Hickling und Mitarbeiter [52] fanden retrospektiv an 50 ARDS-Patienten eine Letalitätsrate von 16\%, die signifikant unter der mit dem APACHE II-Score vorausgesagten von 39,6\% lag. Diese günstigen Ergebnisse konnten die Autoren [51] auch in einer 1994 vorgestellten, prospektiven Studie bestätigen. Lewandowski und Mitarbeiter [68] wiesen an 97 ARDS-Patienten nach, daß durch ein Beatmungskonzept, das druckkontrollierte Beatmung und PHC einschließt, der PIP auf $30-35 \mathrm{cmH}_{2} \mathrm{O}$ gesenkt werden konnte. Es wurden dabei mittlere, maximale $\mathrm{PaCO}_{2}$-Werte von 58-71 mmHg toleriert. Amato und Mitarbeiter [2, 3] zeigten in einer randomisierten, kontrollierten Studie, daß sich in der Gruppe der mit PHC behandelten Patienten die Oxygenation verbesserte, niedrigere PIPs angewendet werden konnten und weniger Patienten an respiratorischer Insuffizienz verstarben.

Bedenken bei der Anwendung der PHC beziehen sich oft auf die langanhaltende, extrazelluläre Azidose, die mit der Hyperkapnie einhergeht. Für die Effekte der akuten Hyperkapnie ist jedoch nicht der extra -, sondern der intrazelluläre $\mathrm{pH}$-Wert entscheidend [109]. Durch intrazelluläre Pufferung, Abbau organischer Säuren und $\mathrm{H}^{+}$-Ionenaustausch über Protonenpumpen der Zellwand normalisiert sich der intrazelluläre $\mathrm{pH}$-Wert innerhalb von 3 Stunden weitgehend [110]. Diese rasche Anpassung erklärt die gute Toleranz der ARDS-Patienten gegenüber der PHC. Die Normalisierung des extrazellulären $\mathrm{pH}$ dauert dagegen meist mehr als 3 Tage. Extrazelluläre Azidosen sollten nur gepuffert werden, wenn die klinischen Auswirkungen der Hyperkapnie dies unbedingt erforderlich machen; weiterhin muß man in einer solchen Situation auch eine „Rücknahme" der PHC durch Vergrößerung des Tidalvolumens oder Modifikation der Beatmungsfrequenz überlegen.

Ein erhöhter, intrakranieller Druck ist eine Kontraindikation für die Anwendung der PHC, da eine Hyperkapnie durch zerebrale Vasodilatation zu Vermehrung des intrazerebralen Blutvolumens und zu einer Zunahme des Hirnödems führt [87]. Ist eine Anwendung der PHC unverzichtbar, sollte der intrakranielle Druck kontinuierlich gemessen werden, damit das Ausmaß der PHC gesteuert werden kann. Hyperkapniebedingte Krampfanfälle treten erst bei exzessiv hohen $\mathrm{PaCO}_{2}$-Werten (150-200 mmHg) auf [50], die unter Anwendung der PHC normalerweise nicht 
erreicht werden. Patienten mit vorbestehenden, epileptischen Erkrankungen, Hirnschäden oder die Krampfschwelle senkender Medikation können auch bei niedrigeren $\mathrm{PaCO}_{2}$-Werten zerebrale Anfälle entwickeln. Das Ausmaß der PHC muß daher bei Auftreten von Krampfanfällen begrenzt werden; vorbestehende Anfallsfleiden stellen eine relative Kontraindikation für die Anwendung der PHC dar.

Eine kardiozirkulatorische Depression bei Hyperkapnie durch systemische Vasodilatation und Abnahme der myokardialen Kontraktilität ist bekannt und kann bei ARDSPatienten mit vorbestehend eingeschränkter Herzfunktion zu einem inadäquat niedrigen Herzminutenvolumen und zu Hypotension führen. Diese Nebenwirkungen der PHC sollten mit differenzierter Volumen- und Katecholamintherapie behandelt werden, in schweren Fällen muß das Ausmaß der PHC begrenzt, bzw. die Anwendung abgebrochen werden.

Arrhythmien unterschiedlicher Ausprägung können bei hyperkapnischen Azidosen [50,96] beobachtet werden, die zum Teil einer erhöhten Katecholaminfreisetzung, zum Teil einer direkten Wirkung der Hyperkapnie auf das Myokard zuzuschreiben sind. Die Anwendung der PHC muß unter Beachtung der Art und Schwere der Herzrhythmusstörungen differenziert und im Ausmaß der Anwendung begrenzt werden.

Die pulmonalarterielle Hypertension ist typisch für das ARDS und mit zunehmendem Schweregrad stärker ausgeprägt. Die Anwendung von PHC kann durch pulmonale Vasokonstriktion zu einer weiteren Steigerung des pulmonalarteriellen Druckes und damit in schweren Fällen zu einer Rechtsherzdekompensation führen [50]. Puybasset und Mitarbeiter [97] konnten aber in einer prospektiven Studie an 11 ARDS-Patienten zeigen, daß sich dieser Effekt durch die inhalative Applikation von NO antagonisieren läßt.

Die durch eine Hyperkapnie ausgelösten Veränderungen der Pharmakokinetik von in Ihrer Wirkung pH-Wert abhängiger Medikamente - insbesondere der Katecholamine - [87] müssen beachtet und die Dosierung der Therapeutika entsprechend angepaßt werden. Dies stellt jedoch keine Kontraindikation für die Anwendung der PHC dar.

\section{Beatmung in Bauchlage (Prone Positioning)}

Lange Phasen der Immobilisation führen bei Intensivpatienten unter anderem zu Decubiti, Venenthrombosen, Embolien, Pneumonien und zu Störungen des Ventilations-/ Perfusions-Verhältnisses durch Atelektasenbildung und Sekretverhalt. Durch häufiges Umlagern des Patienten können diese Komplikationen größtenteils vermieden werden. Fishman [32] forderte für Patienten mit einseitig ausgeprägteren Lungenerkrankungen ,down with the good lung“. Diese Maßnahme kann durch die der Schwerkraft folgende, bessere Durchblutung der unten liegenden, we- niger geschädigten Lunge bei seitendifferenter Ausprägung der Lungenerkrankung zu einer Verbesserung des pulmonalen Gasaustausches und zu einer Erhöhung des $\mathrm{PaO}_{2}$ führen [32, 89, 101].

In den posterobasalen Abschnitten der ARDS-Lunge befinden sich häufig ausgeprägte Atelektasen, die man mit CTUntersuchungen in Rückenlage nachweisen kann [40]. Untersuchungen unterschiedlichen Designs konnten den positiven Effekt der Bauchlagerung (,,prone position“) auf den pulmonalen Gasaustausch zeigen. Es gibt jedoch auch bei diesem Verfahren eine Gruppe, die von der Bauchlage nicht oder nur wenig profitiert (,,non-responder“). Der günstige Einfluß der Lagerungsmaßnahmen auf den Gasaustausch der ARDS-Lunge kann durch Soforteffekte unmittelbar nach Lagewechsel und durch 2 bis 4 Stunden verzögert eintretende Effekte erklärt werden. Zu den Soforteffekten zählt man eine mögliche Umverteilung der Durchblutung in noch gesunde Bezirke (neue Arbeiten widersprechen allerdings dieser Theorie [131]) und das "Rekruitment" von Kompressionsatelektasen. Verzögerte Effekte sind der Anstieg der funktionellen Residualkapazität, die Änderung der Ventilationsverteilung mit Rückverteilung und Wiedereröffnung von atelektatischen Bereichen, die Veränderung des hydrostatischen Druckes und ein dadurch induzierter Rückgang des interstitiellen Lungenödems sowie der verbesserte Abfluß von Bronchialsekret. Damit wird der Konsolidierung der atelektatischen Bereiche entgegengewirkt und das Risiko einer Superinfektion des Gewebes vermindert. Aufgrund dieser positiven Effekte erscheint es bei allen ARDSPatienten gerechtfertigt, einen möglichen Einfluß der Bauchlage auf die Lungenfunktion des Patienten zu überprüfen. Bei einer Verbesserung der Oxygenation sollte die Maßnahme täglich für einige Stunden bis zum Ende der Akutphase durchgeführt werden.

\section{Schlußbemerkung}

Die Vielzahl der experimentellen sowie klinischen Untersuchungen zu den Pathomechanismen und den verschiedenen Therapieansätzen bei der Behandlung des ARDS haben in den letzten Jahren zu einem enormen Erkenntniszuwachs und einem größeren Einblick in das Krankheitsbild selbst geführt. Auch wenn weiterhin von einer hohen Letalitätsrate des ARDS ausgegangen werden muß [6], legt der derzeitige Stand der Erkenntnis nahe, daß die Anwendung und Kombination geeigneter Therapiemaßnahmen, wie z. B. die Begrenzung inspiratorischer Spitzendrücke, die Applikation von PEEP, Bauchlagerung, iNO, Surfactantapplikation und permissive Hyperkapnie einen beatmungsassoziierten Lungenschaden reduzieren und damit das Überleben der Patienten verbessert werden kann.

Danksagung Wir danken Herrn Dr. Weismann, Firma Dräger Lübeck, für die Bereitstellung der Evita II Version 76 und die freundliche Unterstützung bei der Erstellung der Druck-/Volumenkurven. 


\section{Literatur}

1. Albert R, Lakshminarayan S, Hildebrandt J, Kirk W, Butler J (1979) Increased surface tension favors pulmonary edema formation in anesthetized dogs' lungs. J Clin Invest 63:1015-1018

2. Amato M, Barbas C, Medeiros D, Magaldi R, Schettino G, Lorenzi Filho G, Kairalla R, Deheinzelin D, Munoz C, Oliveira R, Takagaki T, Carvalho (1998) C Effect of a protective-ventilation strategy on mortality in the acute respiratory distress syndrome. $\mathrm{N}$ Engl $\mathrm{J}$ Med 338:347-354

3. Amato M, Barbas C, Medeiros D, Schettino G, Lorenzi Filho G, Kairalla R, Deheinzelin D, Morais C, Fernandes E, Takagaki T (1995) Beneficial effects of the "open lung approach" with low distending pressures in acute respiratory distress syndrome. A prospective randomized study on mechanical ventilation. Am J Respir Crit Care Med 152:1835-1846

4. Annest SJ, Gottlieb ME, Rhodes GR, Paloski WH, Barie P, Newell JC, Shah DM (1981) Nitroprusside and nitroglycerine in patients with posttraumatic pulmonary failure. J Trauma 21:1029-1031

5. ARDS-Net Study 01 (1999) Prospective, Randomized, Multi-Center Trial of $12 \mathrm{ml} / \mathrm{kg}$ vs. $6 \mathrm{ml} / \mathrm{kg}$ Tidal Volume Positive Pressure Ventilation for Treatment of Acute Lung Injury and Acute Respiratory Distress Syndrome. http://hedwig.mgh.harvard.edu/ ardsnet/ards01.html

6. Artigas A, Bernard G, Marini J (1998) The American-European Consensus Conference on ARDS, Part 2. Intensive Care Med 24:378-398

7. Ashbaugh D, Bigelow D, Petty T, Levine B (1967) Acute respiratory distress in adults. Lancet II:319-323.

8. Benjamin J, Murtagh P, Proctor D, Menkes H, Permutt S (1974) Pulmonary vascular interdependence in excised dog lobes. J Appl Physiol 37:887-94

9. Bernard G, Artigas A, Brigham K, Carlet J, Falke K, Hudson L, Lamy M, Legall J, Morris A, Spragg R (1994) The American-European consensus conference on ARDS. Definitions, mechanisms, relevant outcomes, and clinical trial coordination. Am J Respir Crit Care Med 149:818-824

10. Bigatello L, Hurford W, Kacmarek R, Roberts J, Zapol W (1994) Prolonged inhalation of low concentrations of nitric oxide in patients with severe adult respiratory distress syndrome. Effects on pulmonary hemodynamics and oxygenation. Anesthesiology 80:761-770
11. Bredenberg C, Nieman G, Paskanik A, Hart A (1986) Microvascular membrane permeability in high surface tension pulmonary edema. J Appl Physiol 60:253-259

12. Brett SJ, Hansell DM, Evans TW (1998) Clinical correlates in acute lung injury: response to inhaled nitric oxide. Chest 114:1397-1404

13. Broccard A, Shapiro R, Schmitz L, Ravenscraft S, Marini J (1997) Influence of prone position on the extent and distribution of lung injury in a high tidal volume oleic acid model of acute respiratory distress syndrome. Crit Care Med 25:16-27

14. Brochard L, Roudot Thoraval F, Roupie E, Delclaux C, Chastre J, Fernandez Mondejar E, Clementi E, Mancebo J, Factor P, Matamis D, Ranieri M, Blanch L, Rodi G, Mentec H, Dreyfuss D, Ferrer M, Brun Buisson C, Tobin M Lemaire F (1998) Tidal volume reduction for prevention of ventilator induced lung injury in acute respiratory distress syndrome. The Multicenter Trail Group on Tidal Volume reduction in ARDS. Am J Respir Crit Care Med 158:1831 -1838

15. Burke W, Nahum A, Ravenscraft $S$, Nakos G, Adams A, Marcy T, Marini J (1993) Modes of tracheal gas insufflation. Comparison of continuous and phase-specific gas injection in normal dogs. Am Rev Respir Dis 148:562-568

16. Carvalho C, Barbas C, Medeiros D, Magaldi R, Lorenzi-Filho G, Kairalla R, Deheinzelin D, Munhoz C, Kaufmann M, Ferreira M, Takagaki T, Amato $\mathrm{M}$ (1997) Temporal hemodynamic effects of permissive hypercapnia associated with ideal PEEP in ARDS. Am J Respir Crit Care Med 156:1458-1466

17. Cereda M, Foti G, Musch G, Sparacino M, Pesenti A (1996) Positive end expiratory pressure prevents the loss of respiratory compliance during low tidal volume ventilation in acute lung injury patients Chest. 109:480-485

18. Chollet Martin S, Gatecel C, Kermarrec N, Gougerot Pocidalo MA, Payen DM (1996) Alveolar neutrophil functions and cytokine levels in patients with the adult respiratory distress syndrome during nitric oxide inhalation. Am J Respir Crit Care Med 153:985-990

19. Clark R, Gerstmann D, Null D, deLemos R (1992) Prospective randomized comparison of high-frequency oscillatory and conventional ventilation in respiratory distress syndrome. Pediatrics 89:5-12
20. Cooper J, van der Zee H, Line B, Malik A (1987) Relationship of end-expiratory pressure, lung volume, and $99 \mathrm{mTc}-$ DTPA clearance. J Appl Physiol 63:1586-15890

21. Corbridge T, Wood L, Crawford G, Chudoba M, Yanos J, Sznajder J (1990) Adverse effects of large tidal volume and low PEEP in canine acid aspiration. Am Rev Respir Dis 142:311-315

22. Croce MA, Fabian TC, Patton JH, Jr., Melton SM, Moore M, Trenthem LL (1998) Partial liquid ventilation decreases the inflammatory response in the alveolar environment of trauma patients. J Trauma 45:273-280

23. Dambrosio M, Roupie E, Mollet J, Anglade M, Vasile N, Lemaire F, Brochard L (1997) Effects of positive end expiratory pressure and different tidal volumes on alveolar recruitment and hyperinflation. Anesthesiology 87:495-503

24. Dellinger R, Zimmerman J, Taylor R, Straube R, Hauser D, Criner G, Davis K, Hyers T, Papadakos P (1998) Effects of inhaled nitric oxide in patients with acute respiratory distress syndrome results of a randomized phase II trial. Inhaled Nitric Oxide in ARDS Study Group Crit Care Med 26:15-23

25. Dreyfuss D, Basset G, Soler P, Saumon G (1985) Intermittend positive-pressure hyperventilation with high inflaltion pressures produces pulmonary microvascular injury in rats. Am Rev Resir Dis 132:880-884

26. Dreyfuss D, Saumon G (1998) Ventilator induced lung injury:lessons from experimental studies. Am J Respir Crit Care Med 157:294-323

27. Dreyfuss D, Soler P, Basset G, Saumon G (1988) High inflation pressure pulmonary oedema:respective effects of high airway pressure, high tidal volume and positive end-expiratory pressure. Am Rev Respir Dis 137 1159-1164

28. Dreyfuss D, Soler P, Saumon G (1992) Spontaneous resolution of pulmonary edema caused by short periods of cyclic overinflation. J Appl Physiol 72:2081-2089

29. Dreyfuss D, Soler P, Saumon G (1995) Mechanical ventilation-induced pulmonary edema. Interaction with previous lung alterations. Am J Respir Crit Care Med 151:1568-1575.

30. Faithfull N (1992) Second generation fluorocarbons. Adv Exp Med Biol 317:441-452

31. Falke K, Pontoppidan H, Kumar A, Leith D, Geffin B, Laver M (1972) Ventilation with end expiratory pressure in acute lung disease. J Clin Invest 51:2315-2323 
32. Fishman A (1981) Down with the good lung. N Engl J Med 304(9):537-538

33. Fort P, Farmer C, Westerman J, Johannigman J, Beninati W, Dolan S, Derdak S (1997) High-frequency oscillatory ventilation for adult respiratory distress syndrome-a pilot study. Crit Care Med 25:937-947

34. Frostell C, Fratacci M, Wain J, Jones R, Zapol W (1991) Inhaled nitric oxide. A selective pulmonary vasodilator reversing hypoxic pulmonary vasoconstriction. Circulation 83:2038-2047

35. Fu Z, Costello M, Tsukimoto K, Prediletto R, Elliot A, Mathieu-Costello O, West J (1992) High lung volume increases stress failure in pulmonary capillaries. J Appl Physiol 73:123-133

36. Fuhrman B, Paczan P, DeFrancisis M (1991) Perfluorocarbon-associated gas exchange. Crit Care Med 19:712-722

37. Gammon B, Shin M, Buchalter S (1992) Pulmonary barotrauma in mechanical ventilation. Patterns and risk factors. Chest 102:568-572

38. Gattinoni L, Pelosi P, Crotti S, Valenza F (1995) Effects of positive end-expiratory pressure on regional distribution of tidal volume and recruitment in adult respiratory distess syndrome. Am J Respir Crit Care Med 151:1807-14

39. Gattinoni L, Pelosi P, Suter PM, Pedoto A, Vercesi P, Lissoni A (1998) Acute respiratory distress syndrome caused by pulmonary and extrapulmonary disease:different syndromes? Am J Respir Crit Care Med 158:3-11

40. Gattinoni L, Pesenti A (1988) Relationships between lung computed tomographic density, gas exchange, and PEEP in acute respiratory failure. Anesthesiology 69:824-832

41. Gattinoni L, Pesenti A, Leonello A, Francesca R, Bombono M (1987) Pressure-volume curve of total respiratory system in acute respiratory failure. Am Rev Respir Dis 136:730-736

42. Gerlach H, Pappert D, Lewandowski K, Rossaint R, Falke KJ (1993) Longterm inhalation with evaluated low doses of nitric oxide for selective improvement of oxygenation in patients with adult respiratory distress syndrome Intensive Care Med 19:443-449

43. Gerlach H, Rossaint R, Pappert D, Falke K (1993) Time-course and doseresponse of nitric oxide inhalation for systemic oxygenation and pulmonary hypertension in patients with adult respiratory distress syndrome. Eur J Clin Invest 23:499-502

44. Ghignone M, Girling L, Prewitt RM (1985) Effects of vasodilators on canine cardiopulmonary function when a decrease in cardiac output complicates an increase in right ventricular afterload. Am Rev Respir Dis 131:527-530
45. Gluck E, Heard S, Patel C, Mohr J, Calkins J, Fink M, Landow L (1993) Use of ultrahigh frequency ventilation in patients with ARDS. A preliminary report. Chest 103:1413-1420

46. Gommers D, Lachmann B (1995) Surfactant therapy in the adult patient. Curr Opinion Crit Care 1:57-61

47. Hafner D, Beume R, Kilian U, Krasznai G, Lachmann B (1995) Dose-response comparisons of five lung surfactant factor (LSF) preparations in an animal model of adult respiratory distress syndrome (ARDS). Br J Pharmacol 115:451-458

48. Hafner D, Germann PG, Hauschke D (1994) Effects of lung surfactant factor (LSF) treatment on gas exchange and histopathological changes in an animal model of adult respiratory distress syndrome (ARDS): comparison of recombinant LSF with bovine LSF. Pulm Pharmacol 7:319-332

49. Hamilton P, Onayemi A, Smyth J, Gillan J, Cutz E, Froese A, Bryan A (1983) Comparison of conventional and high frequency ventilation, oxygenation, and lung pathology. Appl Physiol 5:131-138

50. Hickling K (1992) Low volume ventilation with permissive hypercapnea in the adult respiratory distress syndrome. Clin Intensive Care 3:67-78

51. Hickling K, Henderson S, Jackson R (1990) Low mortality associated with low volume pressure limited ventilation with permissive hypercapnia in severe adult respiratory distress syndrome. Intensive Care Med 16:372-377

52. Hickling K, Walsh J, Henderson S, Jackson R (1994) Low mortality rate in adult respiratory distress syndrome using low-volume, pressure-limited ventilation with permissive hypercapnia: a prospective study. Crit Care Med 22:1568-1578

53. Hirschl R, Parent R, Tooley R, McCracken M, Johnson K, Shaffer T, Wolfson M, Bartlett R (1995) Liquid ventilation improves pulmonary function, gas exchange and lung injury in a model of respiratory failure. Ann Surg 221:79-88

54. Hirschl R, Pranikoff T, Wise C, Overbeck M, Gauger P, Schreiner R, Dechert R, Bartlett R (1996) Initial experience with partial liquid ventilation in adult patients with the acute respiratory distress syndrome JAMA 275:383-389

55. Howard A, Alexander W, Nerem R, Griendling K, Taylor R (1997) Cyclic strain induces an oxidative stress in endothelial cells. Am J Physiol 272:C421-427
56. Kaisers U, Kuhlen R, Keske U, Sommerer A, Mohnhaupt A, Falke K, Rossaint R (1998) Superimposing positive end-expiratory pressure during partial liquid ventilation in experimental lung injury. Eur Respir J 11:1035-1042

57. Kaisers U, Max M, Walter J, Kuhlen R, Pappert D, Falke K, Rossaint R (1997) Partial liquid ventilation with small volumes of FC 3280 increases survival time in experimental ARDS [see comments]. Eur Respir J 10:1955-1961

58. Kirby R, Downs J, Civetta JM, Modell JH, Dannemiller FJ, Klein E, Hodges M (1975) High level positive end-expiratory pressure (PEEP) in acute respiratory insufficiency. Chest 67:156-163

59. Kirmse M, Fujino Y, Hess D, Kacmarek RM (1998) Positive end-expiratory pressure improves gas exchange and pulmonary mechanics during partial liquid ventilation. Am J Respir Crit Care Med 158:1550-1556

60. Koleff M, Schuster D (1995) The acute respiratory distress syndrome. N Eng J Med 323:27-37

61. Kolobow T, Moretti M, Fumagalli R, Mascheroni D, Prato P, Chen V, Jonz M (1987) Severe impariment in lung function induced by high peak airway pressure durin mechanical ventilation: an experimental study. Am Rev Respir Dis 135:312-315

62. Krafft P, Fridrich P, Pernerstorfer T, Fitzgerald R, Koc D, Schneider B, Hammerle A, Steltzer H (1996) The acute respiratory distress syndrome: definitions, severity and clinical outcome. An analysis of 101 clinical investigations. Intensive Care Med 22:519-529

63. Kumar A, Falke K, Geffin B, Aldredge C, Laver M, Lowenstein E, Pontoppidan H (1970) Continuous positivepressure ventilation in acute respiratory failure. N Engl J Med 238:1430-1436

64. Kumar A, Pontoppidan H, Falke KJ, Wilson R, Laver M (1973) Pulmonary barotrauma during mechanical ventilation. Crit Care Med 1:181-6

65. Lachmann B (1992) Open up the lung and keep the lung open. Intensive Care Med 18:319-321

66. Lachmann B, Jonson B, Lindroth M, Robertson B (1982) Modes of artificial ventilation in severe distress syndrome. Crit Care Med 10:724-732

67. Lessard M, Guerot E, Lorino H, Lemaire F, Brochard L (1994) Effects of pressure controlled with different I:E ratios versus volume controlled ventilation on respiratory mechanics, gas exchange, and hemodynamics in patients with adult respiratory distress syndrome. Anesthesiology 80:983-991 
68. Lewandowski K, Pappert D (1995) Permissive hypercapnea in the treatment of ARDS. Am J Respir Crit Care Med 151:A79

69. Lewis J, Jobe A (1993) Surfactant and the adult respiratory distress syndrome. Am Rev Respir Dis 147:218-233

70. Marini J, Kelsen S (1992) Re-targeting ventilatory objectives in adult respiratory distress syndrome. Am Rev Respir Dis 146:2-3

71. Marini J.J, Amato M. B., Lung Recruitment during ARDS in:Acute Lung Injury, Update in Intensive Care and Emergency Medicine Vol. 30 ed. Marini J. J., Evans T. W. New York (Springer) 1998:236-257

72. Marini-J (1994) Ventilation of the acute respiratory distress syndrome. Looking for Mr. Goodmode. Anesthesiology 80:972-975

73. Matamis D, Lemaire F (1984) Total respiratory pressure-volume curves in the adult respiratory distress syndrome. Chest 86:58-66

74. Max M, Kaisers U, Rossaint R (1997) Perspektiven der maschinellen Beatmung im ARDS. Schweiz Med Wochenschr 127:1030-1038

75. McIntyre R, Laws A, Ramachandran $P$ (1969) Positive expiratory pressure plateau: improved gas exchange during mechanical ventilation. Can Anaesth Soc J 16:477-486

76. Miller W, Rice D, Unger K, Bradley B (1981) Effect of PEEP on lung water content in experimental noncardiogenic pulmonary edema. Crit Care Med 9:7-9

77. Morris A, Wallace C, Menlove R, Clemmer T, Orme J, Weaver L, Dean N, Thomas F, East T, Pace N (1994) Randomized clinical trial of pressurecontrolled inverse ratio ventilation and extracorporeal $\mathrm{CO} 2$ removal for adult respiratory distress syndrome. Am J Respir Crit Care Med 149:295-305

78. Muller E, Kolobow T, Mandava S, Jones M, Vitale G, Aprigliano M, Yamada K (1993) How to ventilate lungs as small as $12.5 \%$ of normal: the new technique of intratracheal pulmonary ventilation. Pediatr Res 34:606-610

79. Murray I, Modell J, Gallagher T, Banner M (1984) Titration of PEEP by the arterial minus end tidal carbon dioxide gradient. Chest 85:100-104

80. Muscedere J, Mullen J, Gan K, Slutsky A (1994) Tidal ventilation at low airway pressures can augment lung injury. Am J Respir Crit Care Med 149:1327-1334
81. Nahum A, Burke W, Ravenscraft $S$, Marcy T, Adams A, Crooke P, Marini J (1992) Lung mechanics and gas exchange during pressure-control ventilation in dogs. Augmentation of $\mathrm{CO}_{2}$ elimination by an intratracheal catheter. Am Rev Respir Dis 146:965-973

82. Nahum A, Ravenscraft $S$, Nakos G, Burke W, Adams A, Marcy T, Marini J (1992) Tracheal gas insufflation during pressure-control ventilation. Effect of catheter position, diameter, and flow rate. Am Rev Respir Dis 146:1411-1418

83. Nesti F, Fuhrman B, Steinhorn D, Papo M, Hernan L, Duffy L, Fisher J, Leach C, Paczan P, Burak B (1994) Perfluorocarbon-associated gas exchange in gastric aspiration. Crit Care Med 22:1445-1452

84. Nieman GF, Gatto LA, Paskanik AM, Yang B, Fluck R, Picone A (1996) Surfactant replacement in the treatment of sepsis-induced adult respiratory distress syndrome in pigs. Crit Care Med 24:1025-1033

85. Nishina K, Mikawa K, Takao Y, Obara H (1997) Inhaled nitric oxide does not prevent endotoxin-induced lung injury in rabbits. Acta Anaesthesiol Scand 41:399-407

86. Nolop K, Maxwell D, Royston D, Hughes J (1986) Effect of raised thoracic pressure and volume on $99 \mathrm{mTc}$ DTPA clearance in humans. J Appl Physiol 60:1493-1497

87. Nunn J (1987) The effects of changes in the carbon dioxide tension, Applied respiratory physiology. AnonymousLondon, Butterworths pp 460-470

88. Palmer R, Ferrige A, Moncada S (1987) Nitric oxide release accounts for the biological activity of endothelium-derived relaxing factor. Nature 327:524-526

89. Pappert D, Rossaint R, Slama K, Grüning T, Falke KJ (1994): Influence of positioning on ventilation-perfusion relationships in severe adult respiratory distress syndrome. Chest 106:1511-1516

90. Pardo A, Selman M, Ridge K, Barrios R, Sznajder J (1996):Increased expression of gelatinases and collagenase in rat lungs exposed to $100 \%$ oxygen. Am J Respir Crit Care Med 154:1067-1075

91. Parker J, Hernandez L, Peevy K (1993) Mechanisms of ventilator induced lung injury. Crit Care Med 21:131-43

92. Pelosi P, Cereda M, Foti G, Giacomini M, Pesenti A (1995) Alterations of lung and chest wall mechanics in patients with acute lungy:effects of positive end expiratory pressure. Am J Respir Crit Care Med 152:531-7
93. Pelosi P, D’Andrea L, Vitale G, Pesenti A, Gattinoni L (1994) Vertical gradient of regional inflation in adult respiratory failure. Am J Respir Crit Care Med 149:8-13

94. Pepe P, Potkin R, Reus D, Hudson L, Carrico C (1982) Clinical predictors of the adult respiratory distress syndrome. Am J Surg 144:124-30

95. Picone A, Gatto LA, Nieman GF, Paskanik AM, Lutz C (1996) Pulmonary surfactant function following endotoxin: effects of exogenous surfactant treatment. Shock 5:304-310

96. Price H Lurie A (1958) Role of catecholamines in the initiation of arrhythmic cardiac contraction by carbon dioxide inhalation in anesthetized man. J Pharmac Exp Therap 122:63A

97. Puybasset L, Stewart T, Rouby J, Cluzel P, Mourgeon E, Belin M, Arthaud M, Landault C, Viars P (1994) Inhaled nitric oxide reverses the increase in pulmonary vascular resistance induced by permissive hypercapnia in patients with acute respiratory distress syndrome Anesthesiology 80:1254-1267

98. Ranieri V, Giuliani R, Fiore T, Dambrosio M, Milic Emili J (1994) Volume Pressure Curve of the Respiratory Systems Predicts Effects of PEEP in ARDS: "Occlusion" versus "Constant Flow" Technique. Am J Respir Crit Care Med 149:19-27

99. Ranieri V, Giuliani R, Mascia L, Grasso S, Petruzzelli V, Bruno F, Fiore T, Brienza A (1996) Chest wall and lung contribution to the elastic properties of the respiratory system in patients with chronic obstructive pulmonary disease. Eur Respir J 9:1232-1239

100. Rappaport S, Shpiner R, Yoshihara G, Wright J, Chang P, Abraham E (1994) Randomized, prospective trial of pressure limited versus volume controlled ventilation in severe respiratory failure Crit Care Med 22:22-32

101. Remolina C, Kahn A (1981) Positional hypoxemia in unilateral lung disease. N Engl J Med 304:523-525

102. Rossaint R, Falke K, Lopez F, Slama K, Pison U, Zapol W (1993) Inhaled nitric oxide for the adult respiratry distress syndrome. N Eng J Med 328:399-405

103. Rossaint R, Gerlach H, SchmidtRuhnke H, Pappert D, Lewandowski K, Steudel W, Falke K (1995) Efficacy of inhaled nitric oxide in patients with severe ARDS. Chest 107:1107-1115 
104. Roupie E, Dambrosio M, Servillo G, Mentec H, el Atrous S, Beydon L, Brun-Buisson C, Lemaire F, Brochard L (1995) Titration of tidal volume and induced hypercapnia in acute respiratory distress syndrome. Am J Respir Crit Care Med 152:121-128

105. Sandhar B, Niblett D, Argiras E, Dunnill M, Sykes M (1988) Effects of positive end-expiratory pressure on hyaline membrane formation in a rabbit model of the neonatal respiratory distress syndrome. Intensive Care Med 14:538-546

106. Schmidt G, Hall J (1994) Ventilatory failure, Textbook of respiratory medicine. Edited by J. F.Murray JAN. Philadelphia, Saunders pp 2614-2635

107. Seeger W, Gunther A, Walmrath H, Grimminger F, Lasch H (1993) Alveolar surfactant and adult respiratory distress syndrome. Pathogenetic role and therapeutic prospects. Clin Investig 71:177-190

108. Shapiro B, Cane R, Harrison R (1984) Positive end expiratory pressure therapy in adults with special reference to acute lung injury: A review of the literature and suggested clinical correlations. Crit Care Med 12:127-141

109. Siesjö B (1971) Quantification of $\mathrm{pH}$ regulation in hypercapnia and hypocapnia. Scand J Clin Lab Invest 28:113-119

110. Siesjö B, Folbergrova J (1972) The effect of hypercapnia upon intracellular $\mathrm{pH}$ in the brain, evaluated by the bicarbonate-carbonic acid method and from the creatine phosphokinase equilibrium. J Neurochem 19:2483-2495

111. Sloane P, Gee M, Gottlieb J, Albertine K, Peters S, Burns J, Machiedo G, Fish J (1992) A multicenter registry of patients with acute respiratory distress syndrome. Physiology and outcome. Am Rev Respir Dis 146:419-426

112. Slutsky AS (1993) Mechanical ventilation. Chest 104:1833-1859

113. Smith D, Frankel L, Derish M, Moody R, Black L, Chipps B, Mathers L (1993) High-frequency jet ventilation in children with the adult respiratory distress syndrome complicated by pulmonary barotrauma. Pediatr Pulmonol 15:279-286

114. Stewart T, Meade M, Cook D, Granton J, Hodder R, Lapinsky S, Mazer C, McLean R, Rogovein T, Schouten B, Todd T, Slutsky A (1998) Evaluation of a ventilation strategy to prevent barotrauma in patients at high risk for acute respiratory distress syndrome. Pressure and Volume Limited Ventilation Strategy Group N Engl J Med 338:355-361
115. Suter P, Fairley B, Isenberg M (1975) Optimum end expiratory airway pressure in patients with acute pulmonary failure. N Engl J Med 292(6):284-289

116. Suter PM, Lemaire F, Positive EndExpiratory Pressure in Acute Respiratory Failure in:Physiological Basis of Ventilatory Support, Lung Biology in Health and Disease Vol. 118 ed. Marini JJ, Slutsky AS New York (Marcel Dekker) 1998:873-887

117. Sydow M, Burchardi H, Ephraim E, Zielmann S, Crozier T (1994) Longterm effects of two different ventilatory modes on oxygenation in acute lung injury. Comparison of airway pressure release ventilation and volume-controlled inverse ratio ventilation. Am J Respir Crit Care Med 149:1550-1556

118. Sykes M (1991) Dose mechanical ventilation damage the lungs? Acta Anaesthesiol Scan 35(Supp 95):35-39

119. Sznajder J, Nahum A, Hansen D, Long G, Wood L (1998) Volume recruitment and oxygenation in pulmonary edema:a comparison between HFOV and CMV. J Crit Care 13:126-135

120. The HIFI Study Group (1989) Highfrequency oscillatory ventilation compared with conventional mechanical ventilation in the treatment of respiratory failure in preterm infants. $\mathrm{N}$ Engl J Med 320:88-93

121. Tremblay L, Slutsky A( 1998) Ventilator-induced injury:from barotrauma to biotrauma. Proc-Assoc-Am-Physicians 110:482-488

122. Tremblay L, Valenza F, Ribeiro S, Li J, Slutsky A (1997) Injurious ventilatory strategies increase cytokines and c-fos m-RNA expression in an isolated rat lung model. J Clin Invest 99:944-952

123. Tütüncü A, Akpir K, Mulder P, Erdmann W, Lachmann B (1993) Intratracheal perfluorocarbon administration as an aid in the ventilatory management of respiratory distress syndrome. Anesthesiology 79:1083-1093

124. Tuxen D (1994) Permissive hypercapnia, Principles and practice of mechanical ventilation. Edited by Tobin MJ. New York, McGraw-Hill pp 371-392

125. Uhlig S, Bethmann A (1997) Prolonged hyperventilation is required for release of tumor necrosis factor a but not interleukin- 6 from isolated perfused mouse lung. Am J Respir Crit Care Med 155:A505

126. Verbrugge S, Sorm V, van't Veen A, Mouton J, Gommers D, Lachmann B (1998) Lung overinflation without positive end-expiratory pressure promotes bacteremia after experimental Klebsiella pneumoniae inoculation. Intensive Care Med 24:172-177
127. von Bethmann A, Brasch F, Nusing R, Vogt K, Volk H, Muller K, Wendel A, Uhlig S (1998) Hyperventilation induces release of cytokines from perfused mouse lung. Am J Respir Crit Care Med 157:263-72

128. Walmrath D, Gunther A, Ghofrani H, Schermuly R, Schneider T, Grimminger F, Seeger W (1996) Bronchoscopic surfactant administration in patients with severe adult respiratory distress syndrome and sepsis. Am J Respir Crit Care Med 154:57-62

129. Walmrath D, Schneider T, Pilch J, Schermuly R, Grimminger F, Seeger W (1995) Effects of aerosolized prostacyclin in severe pneumonia. Impact of fibrosis. Am J Respir Crit Care Med 151:724-730

130. Walmrath D, Schneider T, Schermuly R, Olschewski H, Grimminger F, Seeger W (1996) Direct comparison of inhaled nitric oxide and aerosolized prostacyclin in acute respiratory distress syndrome. Am J Respir Crit Care Med 153:991-996

131. Walther S, Domino K, Glenny R, Hlastala M (1999) Positive end-expiratory pressure redistributes perfusion to dependent lung regions in supine but not in prone lambs. Crit Care Med 27:37-45

132. Waters C, Chang J, Gluksberg M, Depaola N, Grotberg J (1997) Mechanical forces alter growth factor release by pleural mesothelial cells. Am J Physiol 272:L522-L557

133. Webb H, Tierney D (1974) Experimental pulmonary edema due to intermittent positive pressure ventilation with high inflation pressures. Protection by positive end-expiratory pressure. Am Rev Respir Dis 110:556-565

134. Zhu GF, Sun B, Niu SF, Cai YY, Lin K, Lindwall R, Robertson B (1998) Combined surfactant therapy and inhaled nitric oxide in rabbits with oleic acid-induced acute respiratory distress syndrome. Am J Respir Crit Care Med 158:437-443 